$\pm N / N$

Global Journals Inc.

की

GLOBAL JOURNAL OF HUMAN-SOCIAL SCIENCE: A

ARTS \& HUMANITIES - PSYCHOLOGY

Volume 20 Issue 10 Version 1.0 Year 2020

Type: Double Blind Peer Reviewed International Research Journal

Publisher: Global Journals

Online ISSN: 2249-460x \& Print ISSN: 0975-587X

\title{
The Being in Becoming or the Becoming-Man of the Son of God: Rahner and the Enunciation of the Logos in Becoming
}

By Andre Luis De Araujo

Universidade Catolica de Pernambuco

Abstract- If discourse analysis fails to account for the infinite realities of the divine Logos, this would not prevent us from trying to discover, within the limits of the analogy, that syntactic operations could correspond to such characteristics of the enunciation of the divine Logos and its self-communication, in the perspectives of Karl Rahner and Joseph Moingt. After all, it will be in the operations of a kind of theological syntax that we will be able to search for such grammar, because these operations undoubtedly assume that theology points to a discourse and, consequently, to the existence of a theological language. A grammar of relations evokes and therefore announces The Being in becoming, revealing a God who speaks and who would not speak through the opacity of Creation if he did not speak, before, in Himself, not to Himself, but to others, in Himself: a true dialogical Logos.

Keywords: the being -the becoming -the son of god -the becoming-man- enunciation prologue of saint john -logos - rahner- moingt - grammar of relations.

GJHSS-A Classification: FOR Code: 130205p

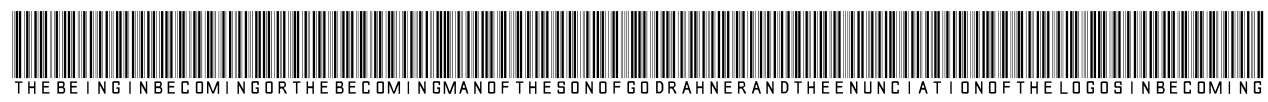

Strictly as per the compliance and regulations of:

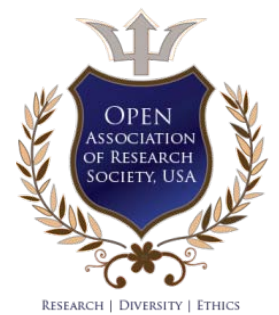

(c) 2020. Andre Luis De Araujo. This is a research/review paper, distributed under the terms of the Creative Commons AttributionNoncommercial 3.0 Unported License http://creativecommons.org/licenses/by-nc/3.0/), permitting all non-commercial use, distribution, and reproduction in any medium, provided the original work is properly cited. 


\title{
The Being in Becoming or the Becoming-Man of the Son of God: Rahner and the Enunciation of the Logos in Becoming
}

\author{
L'Être En Devenir Ou Le Devenir-Homme Du Fils De Dieu : Rahner Et L'énonciation \\ Du Logos En Devenir
}

\author{
Andre Luis De Araujo
}

\begin{abstract}
If discourse analysis fails to account for the infinite realities of the divine Logos, this would not prevent us from trying to discover, within the limits of the analogy, that syntactic operations could correspond to such characteristics of the enunciation of the divine Logos and its selfcommunication, in the perspectives of Karl Rahner and Joseph Moingt. After all, it will be in the operations of a kind of theological syntax that we will be able to search for such grammar, because these operations undoubtedly assume that theology points to a discourse and, consequently, to the existence of a theological language. A grammar of relations evokes and therefore announces The Being in becoming, revealing a God who speaks and who would not speak through the opacity of Creation if he did not speak, before, in Himself, not to Himself, but to others, in Himself: a true dialogical Logos.
\end{abstract}

Keywords: the being -the becoming -the son of god the becoming-man- enunciation - prologue of saint john -logos - rahner- moingt-grammar of relations.

\section{InTRODUCTION}

I e rapport entre Dieu et le monde créé n'est pas simplement un rapport de création. Ce qui détermine cette relation en fin de compte et de façon décisive, selon Karl Rahner, c'est le fait que «Dieu crée le monde non seulement comme ce qui est distinct de lui, mais comme ce à quoi, librement, II veut se communiquer et en son absolue réalité II se communique ». ${ }^{1}$ Cela veut dire que Dieu Lui-même sort de soi et le monde est introduit dans la vie propre de Dieu selon un processus qui ne s'achèvera qu'avec la consommation des siècles. Le théologien dit par là que l'autocommunication de Dieu, désireuse de se répandre en s'extériorisant en amour, constitue à proprement parler le dessein premier de Dieu, bien qu'il reste vrai que Dieu aurait pu créer le monde sans se communiquer à lui.

Dans cette perspective, I'Incarnation se révèle comme le but suprême, de tout temps envisagé, de Author: Universidade Catolica de Pernambuco.

e-mail: aluisaraujosj@gmail.com

${ }^{1}$ RAHNER, K. Marie mère du Seigneur. Paris : Éditions de l'Orante, 1960, p. 18. toute l'œuvre de Dieu se communiquant à son monde. But en fonction duquel tout le reste est tracé, comme condition et comme conséquence. Cette autocommunication de Dieu s'adresse ainsi à toute créature spirituelle. Son fondement propre, sa cime unique, son sceau, sa tangibilité historique comme histoire du salut et son terme irrémédiablement définitif, selon Rahner, elle les trouve dans le fait que Dieu Luimême devient personnellement présent au monde dans I'Incarnation du Logos divin.

De ce fait, dans la quatrième partie de la sixième étape du Traité fondamental de la foi, celle consacrée à Jésus-Christ, Rahner pose bien la question de comment se rendre compte du devenir-homme du Fils de Dieu. D'après lui, bien que nous supposions déjà la foi du christianisme comme donnée par l'annonce kérygmatique des évangiles, la question portant sur la rencontre du Logos divin dans la personne historiquement concrète de Jésus de Nazareth mérite un approfondissement, de par un travail de théologie fondamentale.

En effet, selon Christoph Theobald, les évangiles ne racontent pas seulement l'itinéraire de Jésus, du début jusqu'à la fin de sa vie, mais, en tant que récits de rencontre, ce genre littéraire met l'accent sur la relation d'identification mutuelle établie entre Jésus et ceux et celles qui croisent son chemin. Les évangiles mettent donc en évidence ce qu'il « devient » en et pour ceux et celles dont l'itinéraire croise le sien. ${ }^{2}$ C'est pourquoi il nous semble légitime de nous poser, nous aussi, cette question. Dieu peut-il «devenir » quelque chose? Que voulons-nous dire par le « devenirhomme du Fils de Dieu »? À ce titre, qu'est-ce que nous entendons, en tant que chrétiens, quand nous confessons croire à l'Incarnation du Logos ?

En fait, le chrétien et la philosophie théiste sont ici dans une situation difficile. Ils confessent que Dieu est l'immuable, celui qui « est » purement et simplement.

\footnotetext{
2 Cf. THEOBALD, C. "La réception des Écritures inspirées ». Dans : RSR, 4/2005 (Tome 93), p. 564.
} 
Dieu est l'acte pur qui, dans son invulnérabilité, dans l'absence de manque propre à une réalité infinie, possède depuis toujours, dans une plénitude absolue, immuable et sereine, ce qu'il est, sans aucun devenir, sans même avoir à l'acquérir. De même, la Tradition de l'Église affirme que la nature incréée ignore le mouvement d'où résulterait un changement, une transformation, une altération. Cela dit, est-ce que le devenir-homme du Fils de Dieu, au moment de I'Incarnation, entraînerait, par conséquent, un changement, une transformation ou même une altération à l'Être divin immuable?

À cet égard, nous proposons d'accompagner les mouvements du Logos divin, à l'intérieur du Prologue de l'évangile selon saint Jean. Or, pour ce qui concerne la structure du Prologue johannique, nous remarquons d'abord que le Logos joue son rôle manifestement autour de deux axes : le verbe être et le verbe devenir. Par ailleurs, il est souvent opportun de garder le mot grec logos pour ne rien perdre de la gamme de significations qu'il déploie: "Au temps d'Héraclite, le mot [logos] a d'emblée le sens de discours, récit, parole, mais aussi raison. La référence au langage et discours n'a pas disparu $»^{3}$

Pour cela, dans le premier chapitre de notre analyse, nous essayerons de montrer les mouvements du Logos autour du verbe être et ses implications. Ainsi, bien que l'usage actuel tende à privilégier la traduction de Logos par "Parole", à cause des accointances bibliques de ce terme, nous garderons le mot grec pour maintenir ses différentes nuances afin d'approfondir l'aspect discursif mentionné. En ce sens, prenant en considération le Logos en tant que "discours", en relation étroite avec ses éléments communicationnels les plus simples, c'est-à-dire la présence de celui qui parle, de celui à qui il parle, et d'un système sémiotique qui sert de pont entre les interlocuteurs, nous pouvons nous demander: est-ce que le texte du Prologue du quatrième évangile nous permet de placer une structure discursive à l'intérieur de l'Étre divin? «Au commencement était le Logos, et le Logos était auprès

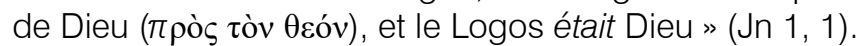
Pouvons-nous ainsi situer en Dieu une structure discursive, voire communicative, avant même de parler de son devenir-homme? De plus, est-ce que cela s'oppose au devenir-homme du Fils de Dieu? Ou bien plutôt cette énonciation de l'Être divin confirme-t-elle et, d'une certaine façon, prépare-t-elle le devenir-homme du Fils de Dieu?

Cela veut dire qu'il faut prendre sérieusement en compte l'analyse du « discours » dans le Prologue, en prenant en considération le Logos en tant que dispositif énonciatif. Car, en effet, il nous semble que c'est tout un dispositif énonciatif qui se manifeste,

${ }^{3}$ JEANNIÈRE, A. « En archê ên o Logos ». Dans : RSR, 2/1995 (Tome 83), p. 244. composé de multiples lignes d'énonciations textuelles, selon divers plans d'organisation discursive. De fait, tout ce qui devient est vie dans le Logos et quand il se tourne vers Dieu, à l'intérieur du Prologue, il est Dieu, mais il est aussi la lumière qui illumine tout homme venant en ce monde, la lumière que les ténèbres n'étouffent pas, mais que les hommes n'ont pas reçue.

Cela signifie qu'il n'est pas suffisant de nous intéresser au devenir-homme du Fils de Dieu. II faut nous occuper d'abord de Celui qui S'énonce, qui parle, de Celui qui est et qui était pour arriver à Celui qui devient, l'Etre en devenir, puisque Dieu Se communique par son Logos, avec lui et en lui. Nous tenterons d'effectuer cette connexion, ce qui nous oblige à prendre le point de vue spécifique de la Trinité immanente, en cherchant à enraciner l'envoi du Logos éternel vers la chair de Jésus.

C'est pourquoi nous ferons intervenir à plusieurs reprises la parole de Joseph Moingt pour parler de cette perspective discursive en Dieu et de son devenir. II nous semble que Rahner lui a donné d'emblée le concept de grammaire avec lequel Moingt fait apparaître une vraie " grammaire de relations ", sans le danger de voir en Dieu trois consciences distinctes, trois centres d'action et, par conséquent, le risque du trithéisme. Pour cette raison, avec Rahner et Joseph Moingt, nous osons dire ainsi que le mystère de Dieu Trinité venant dans le monde s'articule justement à la jonction de sa communication interne et de sa communication externe à d'autres êtres appelés à se mettre en relations les uns avec les autres pour devenir personnes à leur tour.

Pour déployer donc cette "grammaire de relations", nous nous rendons compte que Rahner nous invite d'abord à voir la façon créatrice dont Dieu crée l'homme et l'assume comme son autodiction, comme la grammaire qui rendra possible son autoénonciation. De même, il nous encourage à voir, d'une manière vraiment claire et systématique, l'économie du salut comme voie d'accès au mystère de la Trinité, à partir de l'histoire de la Révélation elle-même. "On pourrait partir de la manière dont Dieu (le Père) se manifeste lui-même, dans l'économie du salut, par la médiation de la Parole et dans l'Esprit, et montrer que cette différence qui apparaît dans 'Dieu pour nous' est celle du 'Dieu en soi' [...] ». ${ }^{4}$

Ce qui se dégage du témoignage de l'Écriture sera donc notre point de départ. En ce sens, Joseph Moingt adopte bien ce registre du langage biblique en tant que mode d'expression de l'économie trinitaire, bien développé par Rahner dans son Traité dogmatique autant que dans la Méditation 12 à propos de I'Incarnation de Dieu. Selon Moingt, Dieu n'est pas solitude, II est tout entier sujet, mais sous le mode de

${ }^{4}$ RAHNER, K. «Le traité dogmatique 'De Trinitate'». Dans: Écrits théologiques, VIII. Paris : DDB, 1967, p. 140. 
l'intersubjectivité.« L'Évangile montre comment Père, Fils et Esprit Saint, en prenant ou en s'adressant mutuellement la parole, se posent comme des personnes, non exactement en face l'une de l'autre, mais plutôt l'une dans l'autre, et chacune de façon différente $»^{5}$ D'après lui, la Trinité devient un circuit des relations, un lieu indissolublement théologique et anthropologique, où le chrétien s'exerce à comprendre ce qu'est la Personne divine, ou plutôt ce qu'elles sont. L'être personne et, par conséquent, le devenir-homme est vécu ainsi dans un jeu de relations à l'autre, c'est-àdire à partir du déploiement d'une vraie « grammaire de relations ».

Après cela, nous serons en condition d'opérer le passage au deuxième chapitre de cette analyse: nous passerons donc de l'Etre au Devenir. Car, le Logos, source de tout commencement possible, devient

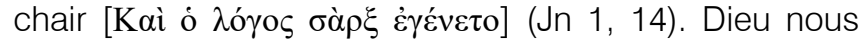
donne Son Logos définitif qui embrasse tout. Et II l'insère réellement dans le monde en sorte qu'll ne peut plus l'en retirer. II l'énonce en insérant, au milieu de ce monde, Son Logos éternel, qui l'exprime Lui-même tout entier, à tel point qu'll est Lui-même devenu, dans la chair de cette humanité, une créature de ce monde. Dieu S'est donné ainsi une réalité définitive, au milieu de ce monde en devenir.

C'est pourquoi, avant d'avancer, il nous faudra faire un petit détour philosophique pour essayer de comprendre le concept du « devenir », ce mot fascinant mais difficile à saisir. Car, malheureusement, il arrive que le devenir se réduise à un mot paradoxalement statique: voir toutes choses en devenir, nous vivons nous-mêmes en devenir. « [...] la pensée se fige sur cet énoncé censé lui apporter le mouvement, et ce qu'on tenait pour son point culminant ressemble fort à un engourdissement: une stase, une extase, une unique masse logique indifférenciée, uniforme et sans promesses ". ${ }^{6}$ En effet, malgré leurs efforts, des philosophes comme Gilles Deleuze et Félix Guattari n'ont pu empêcher que faux amis et détracteurs se liguent pour noyer le concept sous les malentendus: fusion mystique, anthropomorphisme.

De toute façon, considère Rahner, il se trouve qu'il existe un devenir. Et ce n'est pas seulement un fait d'expérience, mais aussi un axiome fondamental de la théologie elle-même, autrement, la liberté, la responsabilité et l'accomplissement de l'homme par sa propre action responsable n'ont aucun sens. Ainsi, d'après lui, le devenir, dans son essence vraie, ne peut être compris comme un simple devenir autre, en tant

\footnotetext{
${ }^{5}$ MOINGT, J. «Dieu qui vient à I'homme, t. II-2 : De l'apparition à la naissance de Dieu - 1. Apparition ». Dans : Cogitatio Fidei, 245. Paris : CERF, 2005, p. 185.

${ }^{6}$ ZOURABICHVILI, F. Conférence prononcée à Horlieu (Lyon) le 27 mars 1997. «Qu'est-ce qu'un devenir, pour Gilles Deleuze?» Document accessible à l'adresse : horlieu-editions.com/brochures/ zourabichvili-qu-est-ce-qu-un-devenir-pour-gilles-deleuze.pdf
}

qu'une réalité devient non pas plus qu'elle n'est, mais autre. Le devenir doit être entendu comme un devenirplus, comme un surgir de plus de réalité, comme atteinte conclue d'une plus grande plénitude d'être ${ }^{7}-$ d'où son concept du devenir-homme du Fils de Dieu. C'est pourquoi ce plus ne saurait être pensé comme simplement ajouté à ce discours qui le précède ; il doit aussi s'affirmer, d'un côté, comme ce qui est effectué par ce qui précède justement, et, d'un autre côté, comme sa croissance en être propre et intérieur.

En définitive, selon Rahner, cela signifie que le devenir, pour être réellement pris au sérieux, doit être nécessairement compris comme autotranscendance réelle, autodépassement de l'être vers son accomplissement. À cet effet, avec le développement du troisième et dernier chapitre de cette analyse - le devenir homme de l'homme - nous souhaitons que l'homme ne reçoive pas simplement cette nouvelle réalité de façon passive, comme effectuée uniquement par Dieu. Mais que la force de ce Logos, parole créatrice, éveille en lui l'image du Dieu invisible, de manière à ce que cette image prenne forme en lui, pour qu'à son tour, il se transforme, jusqu'à devenir ce qu'il était à l'origine.

Rahner nous fait remarquer ainsi qu'il ne s'agit pas de prouver le sens de ce devenir-homme par les déclarations du Magistère officiel de l'Église, bien que l'Église et sa foi soient toujours notre point de départ. Quiconque se tourne vers une véritable compréhension du Mystère doit, dans un mouvement spirituel, prendre distance vis-à-vis des formules dogmatiques et, de retour à elles, trouver le sens de ce qu'il a compris dans son parcours existentiel et herméneutique. II nous semble qu'avant tout une certaine ouverture au Mystère et à sa dynamique propre est la condition de possibilité d'une saisie compréhensive de ce Tout singulier et originaire en devenir en nous et pour nous.

Dans l'histoire de cette autocommunication, c'est l'humanité tout entière, dans son unité, qui se trouve devant Dieu dans la situation d'interpellée, et Jésus-Christ nous invite à une compréhension beaucoup plus radicale de notre vie humaine. Ce que Jésus révèle à l'homme c'est qu'il ne peut être lui-même qu'en se dépouillant de lui-même, qu'en se décentrant par un acte d'amour. Et cet Amour énoncé dans l'Évangile relève moins des comportements individuels que d'un rapport nouveau instauré entre les hommes par la relation de Jésus avec son Père et avec nous.

Jésus se présente ainsi comme l'homme pour et avec les autres. II se présente finalement comme le signe même de l'Amour aux yeux de l'humanité, ce qui est à la racine de lui-même: I'homme pour un Autre. Effectivement, cette histoire se réalise toujours et partout par l'offre de la grâce aux hommes libres de tous les

\footnotetext{
${ }^{7}$ Cf. RAHNER, K. « Jésus Christ ». Dans : Traité fondamental de la foi.
} Paris : Centurion, 1983, p. 212. 
temps et de toutes les conditions. Ainsi, la grâce de Dieu universellement à l'œuvre présente une tangibilité historique (histoire du salut, au sens strict du terme) lorsque, en certains points déterminés de l'espace et du temps, et du fait de leur enchaînement, Dieu, par la révélation de sa Parole atteste sa volonté de sauver le monde.

En guise de conclusion, dans une liberté qui est le fruit de la grâce, l'homme rencontre ce Don du Dieu éternel qui est Dieu Lui-même. II le rencontre avec son corps, son âme, son langage et avec toutes les puissances de son être tout entier, avec tout ce qu'il est, son apport discursif et existentiel, tout ce qu'il a, avec tout ce qu'il fait et tout ce qu'il souffre. Cet accueil de Dieu englobe ainsi tout l'être de l'homme et toute son histoire, pour l'introduire dans la vie éternelle. D'autre part, la grâce sanctifiante signifie, au plus profond, Dieu Lui-même, ses communications à l'esprit créé, le don qu'll est Lui-même. La grâce est aussi lumière, vie, ouverture de la vie spirituelle et personnelle de l'homme aux domaines infinis de Dieu. Elle signifie liberté, force, activité de l'Esprit (Personne divine dans les profondeurs de l'homme), adoption filiale et héritage de la vie éternelle. De telle sorte que, pour nous, tout se ramène à devenir ce que nous sommes, à entendre l'appel que cette grâce fait retentir pour nous et à réaliser librement la fin qui correspond au commencement établi par Dieu : le devenir homme de l'homme à la lumière du devenir-homme du Logos éternel.

\section{L'être en Devenir ou le DeVenir-} HOMME DU FiLS DE DiEU :

RAHNER ET L'ÉNONCIATION DU LOGOS EN

\section{DEVENIR}

a) L'Être

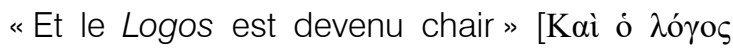

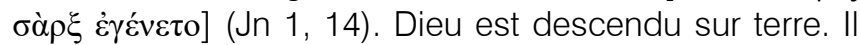
est là comme nous sommes là, dans une semblable appartenance au monde et au temps. Dieu a assumé une nature humaine essentiellement ouverte - affirme Rahner. D'après lui : "L'homme est visité par l'Infini et devient de cette façon celui qui ne s'invente pas luimême, parce que la finitude humaine ne peut être dépassée qu'en s'enfonçant dans la plénitude incompréhensible de Dieu ${ }^{8}{ }^{8}$ La nature humaine est ainsi un chemin à parcourir. De même, la nature divine est un parcours à découvrir.

Arrêtons-nous donc à la singularité de cette révélation pour laisser se dévoiler la nouveauté d'une telle affirmation. Car, si d'une part il reste vrai que «le Logos est devenu chair », d'autre part, confesser un

\footnotetext{
${ }^{8}$ RAHNER, K. « Méditation 12 : L'Incarnation de Dieu ». Dans : L'Esprit Ignatien. (Sous la direction de Michel Fédou). Paris: CERF, 2016, p. 169.
}

Dieu immuable et sans devenir, d'une plénitude éternelle et comblée, n'est pas seulement un postulat de la philosophie, mais une telle confession est aussi un dogme de foi. Comment tenir tout cela ensemble? En effet, être vraiment chrétien exige de creuser au plus profond pour aller jusqu'au bout de cet énoncé. II faut donc que chacun de nous s'ouvre au mystère insurpassable, puisqu'il est la condition de possibilité pour comprendre le destin de l'incompréhensible parole de Dieu qui vient nous saisir.

Parvenues à ce point, la théologie traditionnelle et la philosophie commencent à balbutier, admet Rahner. Elles expliquent que le devenir et le changement seraient du côté de la réalité créée qui est assumée, et non du côté du Logos. En ce sens, le Logos assumerait sans modification ce qui, en tant que réalité créée, a un devenir et le garde même après avoir été assumé et ainsi tout le devenir et toute l'histoire resteraient de ce côté-ci de l'abîme absolu qui sépare, sans confusion, le Dieu immuable et nécessaire du monde changeant et contingent. Pourtant, dit-il :

[...] il reste vrai que le Logos devint homme, que le devenir historique de cette réalité humaine a été sa propre histoire, notre temps, le temps Éternel, notre mort, la mort du Dieu immortel lui-même, et que si l'on répartit sur ces deux réalités - à savoir la parole divine et la nature humaine créée - ces prédicats, apparemment contraditoires et dont une partie semble ne pas pouvoir s'appliquer à Dieu, on ne peut pas oublier que l'une de ces réalités (à savoir la réalité créée) est justement celle du Logos divin lui-même ; donc, après cet expédient d'une répartition qui visait à la résoudre, toute la question est à reprendre à neuf. ${ }^{9}$

Pour Rahner, il s'agit en fait de comprendre que l'affirmation de l'immutabilité de Dieu ne doit pas nous faire perdre de vue que ce qui s'est produit, en Jésus de Nazareth, en fait de devenir et d'histoire, est précisément I'histoire du Logos de Dieu Lui-même, son propore devenir à lui. Pour cette raison, l'intérêt de cette réflexion est de donner à l'Incarnation sa pleine signification: c'est un événement qui survient en Dieu Lui-même. ${ }^{10}$

Cela veut dire que pour parvenir à cette singularité, il importe de ne pas enfermer la révélation de Dieu dans l'événement de la mort et de la résurrection de Jésus. II faut déployer la totalité de cet événement afin de «lui laisser prendre ses pleines dimensions en amont et en aval: en amont, en direction, non seulement de son commencement historique [...], mais encore du commencement absolu du temps [...] ; en aval, en direction de la fin des temps,

\footnotetext{
${ }^{9}$ lbidem, p. 172.

${ }^{10}$ Une telle compréhension, suppose aussi que nous ne considérions pas l'Incarnation comme l'achèvement absolu de la révélation de Dieu, que nous n'arrêtions pas non plus celle-ci à la mort et à la résurrection de Jésus, mais que nous la prolongions jusqu'à son terme, qui est le don du Saint-Esprit répandu « en toute chair », par qui Dieu reste avec nous et en nous jusqu'à la fin du monde. (Cf. MOINGT, J. « Dieu qui vient à l'homme, t. II-2 », pp. 396-397).
} 
mais sans la faire imploser en éternité sous couleur d'eschatologie'» - comme l'affirme encore Joseph Moingt. ${ }^{11}$ Ainsi, d'après lui, connaître le Christ, selon la chair, c'est lui donner ses pleines dimensions d'homme, premier-né des créatures et premier-né d'entre les morts (Col 1, 15-20). Saint Paul nous apprend par là que Jésus ayant traversé victorieusement la mort, en se livrant à elle pour nous, a transgressé les limites d'une existence individuelle et est parvenu à une plénitude d'humanité universelle, celle que nous lui reconnaissons sous le nom de Christ. Celle-ci est précisément où se revèle l'humanité de Dieu, qui n'est rien d'autre que l'être là de Dieu dans le temps propulsé par la venue de son Logos vers la chair.

L'Incarnation concerne donc le Père autant que le Fils, son Logos divin, en lien d'amour indivisible de leur existence dans l'action de l'Esprit Saint. À cet effet, chacun tire de l'autre, sans inégalité, la raison d'être qui il est, amour qui se donne et amour rendu et reçu, amour qui s'interpose, échangé et partagé, comme principe irréductible de l'un à l'autre au sein d'une parfaite communion.

"Et le Logos est devenu chair ». Or, dans cet acte d'Incarnation du Logos, il arrive au Père également de devenir chair, c'est-à-dire de devenir autre, à savoir Père d'un homme et en lui, potentiellement, Père des hommes. II faut oser penser à cela quand nous considérons cet énoncé - insiste Joseph Moingt. Et pour le comprendre, nous pouvons mettre l'accent tantôt sur le sujet divin, tantôt sur la chair de l'homme. Effectivement, nous pouvons opérer des choix ou des soulignements, soit sur le Logos divin, soit sur le prédicat humain. Dès lors, dans cette énonciation du Logos en devenir, c'est la christologie qui devient le terme et le commencement d'une anthropologie, justement en ce qu'elle a de plus radical, comme l'affirme Rahner: "cette anthropologie est, pour l'éternité, théo-logie " ${ }^{12}$ Selon Rahner, elle est la théologie que Dieu Lui-même a formulé en proférant son Logos comme notre chair dans le vide du non-Dieu et du péché.

En d'autres termes, le Logos s'est fait homme. Mais, pour l'instant, nous ne posons pas encore la question de savoir ce que nous entendons par ce devenir-homme qui occupe la sixième étape du Traité fondamental de la foi. De fait, nous pourrions commencer par le mot «homme », peut-être le terme le plus facile à comprendre dans cette proposition. Car homme, c'est ce que nous sommes, ce que nous connaissons de l'intérieur (en chacun de nous-mêmes) et de l'extérieur (à partir du monde qui nous entoure). Pourtant une définition de l'homme est-elle possible?

II paraît que définir, "donner une formule qui circonscrive et énumère adéquatement la somme des

\footnotetext{
${ }^{11}$ Ibidem, pp. 396.

${ }^{12}$ RAHNER, K. « Méditation 12 : L'Incarnation de Dieu », p. 175.
}

éléments, nous ne le pouvons évidemment que pour un objet réel composé d'éléments qui soient à leur tour eux-mêmes des grandeurs intelligibles par soi, donc déjà circonscrites et délimitées par elles-mêmes ${ }^{13}$ considère Rahner à propos de la nature humaine. De ce fait, il nous semble que bien que nous puissions dire globalement quelque chose de valable pour définir notre nature humaine, nous n'avons encore rien dit de décisif sur nous, sauf que l'homme est, dans son essence et par nature, un mystère. Non qu'en lui-même l'homme soit la plénitude infinie et inépuisable du mystère qui nous concerne. C'est bien plutôt parce que, dans son essence propre, dans sa nature, lui vient la conscience de sa faiblesse et, en conséquence, de sa finitude.

De toute façon, nous sommes les signes du Dieu incompréhensible, car "Dieu créa l'homme à son image, à l'image de Dieu il le créa »(Gn 1, 27). Mais cette signification, qui est notre nature même, selon Rahner, n'est entendue et comprise que si nous nous laissons librement saisir par l'Incompréhensible dans le consentement à cet acte qui, au-delà de l'exprimable, est la condition de possibilité d'un discours capable de comprendre quelque chose de ce mystère. L'acceptation ou le refus du mystère que nous sommes, en tant que signes du Mystère de la plénitude, fait toute notre existence. C'est pourquoi nous allons commencer par le sujet de cette proposition : l'énonciation de l'Être divin, le Logos. Et, pour cela, nous proposons d'accompagner les mouvements du Logos, à l'intérieur du Prologue de l'évangile selon saint Jean.

\section{i. Le Logos : un dispositif énonciatif}

Le Logos occupe dans la Tradition et dans l'histoire des dogmes une place considérable. Toutefois, le fait qu'il ne soit nulle part ailleurs mentionné dans l'Évangile est troublant - affirme Joseph Moingt. ${ }^{14}$ En effet, nous ne le trouvons, comme nom propre et personnel du Christ, que dans le Prologue du quatrième évangile. D'une certaine manière, cela nous permet de reconnaître que ce terme, favorisé par la philosophie de l'époque, a été choisi pour introduire cet évangile dans les milieux cultivés. Quoi qu'il en soit, que le nom Logos soit si rare et exclusif dans le Nouveau Testament, du fait qu'il est ignoré des autres évangelistes et de saint Paul et qu'il est culturellement si marqué, cela n'empêche pas qu'il a été très tôt et unanimement accepté par la tradition chrétienne, identifié dans la sémantique biblique comme Parole de Dieu, Verbe éternel du Père: Logos consacré par le dogme et la prédication de l'Église, en référence explicite à la deuxième personne de la Trinité - le Christ.

Cela dit, les modes d'exister du Christ, en Dieu et dans l'histoire, montrent, d'une part, qu'il prend son origine, en tant que Logos, dans l'éternité de Dieu, et que cette origine divine, d'autre part, l'appelle

\footnotetext{
${ }^{13}$ Ibidem, p. 166.

${ }^{14}$ Cf. MOINGT, J. « Dieu qui vient à l'homme, t. II-2 », p. 123.
} 
éternellement à assumer, depuis le commencement, l'histoire de l'humanité qu'il devait assumer dans le temps. Cela nous oblige dès maintenant à ramener ces deux volets de l'existence du Christ afin de voir d'abord l'unité de sa personne, avant même de considérer l'énonciation du Logos divin. Car la dimension de la présence du Logos avant l'Incarnation n'est autre que la présence du Christ dans l'histoire.

Cela signifie que le Logos qui est en Dieu est projeté dans l'histoire «dès avant la fondation du monde ", qu'il la met en marche en direction de son Incarnation, que ce lien dynamique à l'histoire le tient en relation avec l'être humain qu'il doit assumer, et qu'il prépare sa naissance dans le temps en venant au monde depuis le commencement du temps. ${ }^{15}$

La créature peut ainsi, grâce à sa nature la plus intime, être assumée et devenir la matière d'une éventuelle histoire de Dieu. «En créant, Dieu projette toujours la créature comme la grammaire qui permettrait de déchiffrer son propre Nom, s'il le disait, et il ne peut la créer autrement, même s'il se tait, parce que ce silence même suppose toujours des oreilles qui écoutent le mutisme de Dieu $»{ }^{16}$ Dans cette grammaire évoquée par Rahner, nous parviendrons à comprendre que c'est précisement le Logos divin qui est devenu homme, et que lui seul peut le devenir, car si Dieu prononce son propre Nom, dans l'immanence de sa plénitude éternelle, comme le dit Rahner, II le prononce hors de lui. C'est la condition de la simple existence de l'autre, de ce qui est différent de Dieu. De plus, Rahner ajoute :

Dieu, qui est sans origine, se dit lui-même en lui-même, et pour lui-même, et pose ainsi en Dieu même la distinction d'origine des Personnes divines. Et quand Dieu s'exprime lui-même comme tel dans le vide, il profère sa Parole immanente et non une parole quelconque qui pourrait aussi bien convenir à une autre Personne divine. ${ }^{17}$

Ainsi, parce que Dieu Lui-même un jour a proféré cette Parole, son Logos divin, nous supposons par là qu'll a voulu se proférer Lui-même. En conséquence, Rahner se pose la question : "comment pouvait-il le proférer autrement qu'en créant une capacité de percevoir intérieurement cette parole, et qu'en disant de fait sa parole à l'être ainsi ouvert, de sorte que ne fassent plus qu'un l'auto-expression de Dieu et ce qui peut la percevoir ? ${ }^{18}$ Cependant, il admet aussi que si cela advient vraiment, c'est tout à fait un mystère. D'autre part, un mystère est quelque chose d'inattendu, qui nous plonge dans un étonnement et en même temps dans quelque chose d'évident - considère-t-il.

\footnotetext{
${ }^{15}$ MOINGT, J. «L'homme qui venait de Dieu ». Dans : Cogitatio Fidei, 176. Paris: CERF, 1999, p. 673.

${ }^{16}$ RAHNER, K. « Méditation 12 : L'Incarnation de Dieu », p. 173.

17 Ibidem, p. 174.

${ }^{18}$ Ibidem, p. 179
}

L'Incarnation du Logos est pour cela le mystère absolu et pourtant évident. Cela veut dire qu'il faut ainsi chercher les traces de ce Logos. Tout d'abord, comment pouvons-nous traduire ce Logos, un des premiers mots du texte du Prologue de l'évangile selon saint Jean et un des plus importants?

Selon Abel Jeannière, au temps d'Héraclite, le terme a le sens de discours, récit, parole, mais aussi raison. ${ }^{19}$ II nous semble que la référence au langage et au discours n'a donc pas disparu. Mais est-ce que cela nous autorise à situer en Dieu une structure discursive?

Pour le théologien Georges Tavard, la réponse est affirmative. Selon l'auteur, l'usage du même mot (Logos) pour désigner la révélation ou la raison qui sous-tend un discours appartient lui-même à la logique discursive. II soutient que parmi ces sens du mot, qui sont d'ailleurs en corrélation, à savoir - discours, récit, parole, raison -, la tradition théologique en a retenu trois : "Elle [la tradition] a réfléchi sur la deuxième Personne comme Parole unique parlée par Dieu de toute éternité, qui contient en elle-même aussi bien la raison ultime de toutes choses que la possibilité de la révélation de cette ultime raison à travers les paroles temporelles des prophètes $" .^{20}$ Pourtant cela veut-il dire que nous pouvons traduire le Logos énoncé dans le Prologue de l'évangile selon saint Jean par discours? Le quatrième évangile aura-t-il retenu cette nuance discursive, voire communicative, attribuée au mot Logos depuis Héraclite?

Tout bien considéré, nous espérons que l'analyse du discours du Prologue pourra nous aider à mieux saisir ces questions. De même, que le découpage du texte que nous proposons ci-dessous pourra nous donner quelques pistes aussi bien que des éléments de réflexion pour franchir ce terrain.

1. «1. Au commencement était le Logos et le Logos était auprès de Dieu et le Logos était Dieu. 2. II était au commencement auprès de Dieu. 3. Tout fut par lui, et sans lui rien ne fut. 4. Ce qui fut en lui était la vie, et la vie était la lumière des hommes, 5 . et la lumière luit dans le ténèbres et les ténèbres ne l'ont pas saisie.

2. 6. II y eut un homme envoyé de Dieu ; son nom était Jean. 7. II vint pour témoigner, pour rendre témoignage à la lumière, afin que tous crussent par lui. 8. Celui-là n'était pas la lumière, mais il avait à rendre témoignage à la lumière.

3. 9. II était la lumière véritable, qui éclaire tout homme, venant dans le monde. 10. II était dans le monde, et le monde fut par lui, et le monde ne l'a pas reconnu. 11. II est venu chez lui, et les siens ne l'ont pas accueilli.

\footnotetext{
${ }^{19}$ Cf. JEANNIÈRE, A. « En archê ên o Logos », p. 244.

20 TAVARD, G. "Le discours». Dans: La Vision de la Trinité. Paris : CERF, 1989, p. 137.
} 
12. Mais à tous ceux qui l'ont accueilli, il a donné pouvoir de devenir enfants de Dieu, à ceux qui croient en son nom, 13. eux qui ne furent engendrés ni du sang, ni d'un vouloir de chair, ni d'un vouloir d'homme, mais de Dieu.

14. Et le Logos s'est fait chair et il a campé parmi nous, et nous avons contemplé sa gloire, gloire qu'il tient du Père comme Unique-Engendré, plein de grâce et de vérité.

4. 15. Jean lui rend témoignage et s'écrie: "C'est de lui que j'ai dit: Celui qui vient derrière moi, le voilà passé devant moi, parce qu'avant moi il était. "

5. 16. Oui, de sa plénitude nous avons tous reçu, et grâce pour grâce. 17. Car la Loi fut donnée par l'entremise de Moïse, la grâce et la vérité advinrent par l'entremise de Jésus-Christ. 18. Nul n'a jamais vu Dieu; le Fils Unique-Engendré, qui est dans le sein du Père, lui, l'a fait connaître. "

En commençant cette analyse et en regardant de plus près le Prologue, le texte johannique nous fait remarquer, au tout début, une communication interne à Dieu Lui-même. Le Logos divin se présente actif depuis le commencement. II est Dieu et, en même temps, il était auprès de Dieu en façonnant le monde : "Au commencement était le Logos et le Logos était auprès

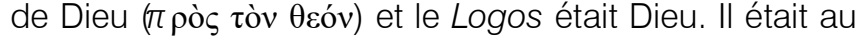
commencement auprès de Dieu » (Jn 1, 1-2). De plus, il nous semble qu'un discours est adressé à quelqu'un, car le texte présentera par la suite une direction et une destination claire (tout homme, le monde, les siens) : « II [le Logos] était la lumière véritable, qui éclaire tout homme, venant dans le monde. II était dans le monde, et le monde fut par lui, et le monde ne l'a pas reconnu. II est venu chez lui, et les siens ne l'ont pas accueilli » (Jn 1, 9-11).

Et bien que le monde ne l'ait pas reconnu ni accueilli, aucun élément de ce monde n'échappera au

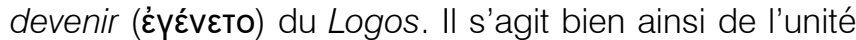
du dessein de Dieu depuis la Création («Tout fut par lui, et sans lui rien ne fut »-Jn 1, 3), comme dans l'histoire du monde (« II était dans le monde, et le monde fut par lui $[\ldots] »-J n 1,10)$. Tout ce mouvement culmine dans le Christ («Et le Logos s'est fait chair et il a campé parmi nous, et nous avons contemplé sa gloire, gloire qu'il tient du Père comme Unique-Engendré, plein de grâce et de vérité »- Jn 1, 14), de telle sorte que chacun de nous qui l'a accueilli se sent concerné, du fait de croire en Lui (« Mais à tous ceux qui l'ont accueilli, il a donné pouvoir de devenir enfants de Dieu, à ceux qui croient en son nom, eux qui ne furent engendrés ni du sang, ni d'un vouloir de chair, ni d'un vouloir d'homme, mais de Dieu »- Jn 1, 12-13).

Et le Logos, présent dès l'origine, s'est fait chair. Ce Logos se révèle ainsi pour être compris et reçu. Mais «[...] le monde ne l'a pas reconnu. II est venu chez lui, et les siens ne l'ont pas accueilli » (Jn 1,
10-11).Malgré cela, aux versets suivants, s'ouvrira une nouvelle perspective à tous ceux qui l'ont accueilli, c'està-dire: Le Logos éternel, Lui-même, leur donnera le pouvoir de devenir enfants de Dieu, à ceux qui croient en son nom(Jn 1, 12). Eux qui ne furent engendrés ni du sang, ni d'un vouloir de chair, ni d'un vouloir d'homme, mais de Dieu auront pontentiellement la force de ce devenir, avant même que le texte nous annonce le Logos devenu chair (Jn 1, 14).

Cela veut dire qu'il y a un véritable tournant, juste au milieu du Prologue, précisément aux versets 12 et 13, introduit même avant I'Incarnation proprement dite, ce qui se manifestera littéralement seulement au verset 14. Ce tournant ouvre par là une étonnante possibilité à l'homme, dévoilée par le changement des interlocuteurs ou bien plutôt par une précision discursive: le passage de la troisième personne du singulier (tout homme, le monde), employée d'une façon communicative encore générale; à la troisième personne du pluriel (les siens, ceux, eux), utilisé selon un mode déjà plus spécifique et identifiable; et finalement à la première personne du pluriel (nous). Nous pouvons donc remarquer que cette identité discursive des interlocuteurs devient de plus en plus discernable, car le texte opère un changement de perspective vers un «nous », au verset 14, c'est-à-dire après I'Incarnation du Logos divin. Est-ce là un indice que la venue du Logos dans la chair nous dispose à un «nous ecclésial ? ». Nous y reviendrons.

Pour l'instant, il nous semble fondamental de nuancer le point suivant : nous pouvons devenir enfants de Dieu en recevant la lumière et en croyant au nom du Verbe, sans être chrétien ni croyant de façon explicite en Jésus-Christ. ${ }^{21}$ Effectivement, les Pères de l'Église ont interprété ce passage comme le temps de la révélation faite au peuple juif pour préparer et annoncer la venue du Logos dans la chair. À vrai dire, ils n'ont pas supposé que le rejet du Logos par les Hébreux aurait été total. Ils ont tous admis, au contraire, que les patriarches, les prophètes et en général les « justes » de l'Ancien Testament avaient cru d'avance en lui. Interprétation qui autorise à les inclure au nombre de ceux à qui le Logos a donné le pouvoir de devenir fils de Dieu.

Néanmoins, comme la suite du texte va nous le montrer, seule la foi dans le Logos incarné, donc en la personne de Jésus-Christ, permet de comprendre vraiment et de vivre à pleinement cet enfantement divin. En ce sens, selon Yves Simoens, cela peut s'exprimer de l'intérieur d'une foi ecclésiale dans le Christ, qui seule rend compte d'un tel engendrement-enfantement de Dieu dans le mondeet dans l'histoire. C'est pourquoi,

${ }^{21}$ Cf. LÉON-DUFOUR, X. Lecture de l'évangile selon Jean, t. I (Parole de Dieu). Paris : Seuil, 1988, p. 107 et 148 et SIMOENS, Y. Selon Jean. 2. Une interprétation. Bruxelles : Éditions de l'Institut d'Études Théologiques, 1997, p. 40. 
au sein de cette communauté ecclésiale évoquée par le Prologue, l'identité de chacun se reconnaît, se révèle et s'intègre. Puisque chacun de nous (narrateur et lecteur) possède un visage concret, peut être nommé et se trouve existentiellement impliqué de par son interlocution avec l'Être divin, dans cette communauté rassemblée autour du Logos incarné.

Dans cette perspective, le Logos, en se faisant chair, permet à Dieu de mieux Se communiquer à nous, en vue d'habiter ce monde et la Création. De ce fait, cette structure discursive se trouve amplifiée, comme nous l'avons vu, à partir du texte du Prologue. C'est pourquoi nous la rapprochons maintenant de la notion discursive utilisée par le philosophe Francis Jacques, car il nous semble que le Logos, depuis le commencement, se communique à plusieurs niveaux en dispositif énonciatif. Selon l'auteur, un dispositif énonciatif est un «composé de multiples lignes sur lesquelles se disposent les positions différentielles de l'énonciation, disons les voix énonciatives ". ${ }^{22}$ En effet, un mouvement complexe se profile à l'intérieur du texte johannique, à divers plans d'organisation d'une structure communicationnelle, comme nous venons de le démontrer. Démêler donc les lignes de ce dispositif énonciatif nous a demandé de dresser la carte de cette énonciation discursive sur le terrain textuel, afin de relever les marques d'énonciation du Logos divin avec ses interlocuteurs (tout homme, le monde, les siens, ceux, nous), par un travail qui s'éloigne d'une approche purement statique, pour s'attacher à la production dynamique elle-même : l'Être divin S'énonce en devenir et, par Lui, avec Lui et en Lui, nous devenons enfants de Dieu. À tel point que "nous» (la communauté ecclésiale) contemplons « [...] sa gloire, gloire qu'il tient du Père comme Unique-Engendré, plein de grâce et de vérité » (Jn 1, 14).

En outre, bien que la révélation de Dieu dans son Logos soit adressée à quiconque, à des destinataires eux-mêmes appelés à bénéficier du don de sa vie, très vite se trouve aussi abordé le registre de la liberté humaine, dans le Prologue, en conformité avec toute la tradition biblique. Dieu en créant prend le risque de se voir refusé. Son amour va jusqu'à ce point. Mais la vie ne se laisse pas vaincre par les puissances de la mort et la lumière ne se laissera pas occulter; au contraire, elle sera révélée encore davantage par la ténèbre. Ce refus se trouve ainsi couronné grâce à un acte de croire qui est engendré par Dieu.

En fait, nous ne pouvons faire cette découverte que parce que, de sa plénitude, nous avons vraiment reçu cette grâce : devenir enfants de Dieu, à savoir, ne naître « [...] ni du sang, ni d'un vouloir de chair, ni d'un vouloir d'homme, mais de Dieu» (Jn 1, 13). Être engendré par Dieu, de son Évangile, né d'en haut, né

\footnotetext{
${ }^{22}$ JACQUES, F. « La parole et l'écriture ». Dans : L'arbre du texte et ses possibles. Paris : Librairie Philosophique J.Vrin, 2007, p. 89.
}

de son Esprit.«Or, cette naissance d'en haut n'est possible que parce que Dieu fait connaître ce qu'll est en Lui-même, par sa Parole faite chair $"{ }^{23}$

Finalement, compte tenu de cette analyse discursive, avec Georges Tavard, nous pouvons en retenir que le mot Logos, situé au cœur du Prologue de l'évangile selon saint Jean, peut être compris comme Discours plutôt que comme simple ingrédient d'un discours quelconque. Néanmoins, quand Tavard affirme que «Traduire Logos par Discours serait donc plus correct que de le traduire par Parole ou Verbe ", ${ }^{24}$ cela nous semble trop exclusif, car le mot grec Logos garde une richesse sémantique qu'il faut maintenir. C'est-àdire: du Logos peuvent jaillir des nuances actives et subjectives. Ainsi, si d'une part nous le traduisons par Verbe, il peut exprimer des actions verbales performatives, en tant qu'il est le Logos divin qui façonne le monde. D'autre part, si nous le traduisons par Parole, le Logos conserve donc ses traces subjectives, à savoir, les implications d'un sujet présent auprès du Père.

Cela veut dire que nous ne pouvons pas limiter les attributions de l'Être divin. En même temps, nous ne voulons pas pour autant nier que le Logos en tant que discours «implique vraiment une structure de communication entre ceux qui parlent et ceux qui écoutent $"{ }^{25}$ Au contraire, quand nous admettons, sans aucun risque, la traduction du grec: Logos = Discours et que nous l'appliquons au texte du Prologue: " $\mathrm{Au}$ commencement était le Discours, et le Discours était auprès de Dieu, et le Discours était Dieu » (cf. Jn 1, 1), les notions relatives à Parole et Verbe se trouvent bien sauvegardées, car il s'agit clairement de la Parole ou du Verbe de Dieu qui habite le Discours divin.

Par ailleurs, nous n'oublions pas non plus que le Logos dans le Prologue de l'évangile selon saint Jean se comporte en termes d'un dispositif énonciatif. Cela préserve tout un dynamisme d'énonciation active (objective/performative) et subjective attribué au Logos divin, à plusieurs niveaux discursifs, depuis le commencement. Car, à vrai dire, tout discours est composé a priori de paroles et de verbes. Autrement dit, cette structure discursive, à la fois subjective (le sujet) et objective (le verbe), inhérente au Logos, permet la réalisation de tout énoncé du langage, du fait que le Logos assume la totalité des énoncés linguistiques, parce qu'il est sujet (Parole) et parce qu'il est prédicat (Verbe).

À cet effet, cette Parole incarnée n'est pas simplement une parole quelconque, comme le disait Rahner, au tout début de cette analyse. Elle est aussi le

\footnotetext{
23 THEOBALD, C. Croire aujourd'hui en Jésus, Christ et Saint de Dieu, 2016, p. 83. (Cours de Christologie - Polycopié)

${ }^{24}$ TAVARD, G. «Le discours ». Dans : La Vision de la Trinité. pp. 137138.

${ }^{25}$ Ibidem, p. 138.
} 
Verbe de Dieu, son vrai Discours, capable d'animer à la fois l'énonciation divine et humaine, puisque « le Logos s'est fair chair ».Cette énonciation du Logos divin en devenir rend possible le langage, du fait qu'il était présent au commencement dans la parole créatrice qui façonne le monde, de même qu'il était auprès de Dieu, qu'il est Dieu et qu'il s'est fait capable d'assumer, par son Incarnation, les deux côtés de cette proposition : il est vraiment le sujet divin et le prédicat humain, puisqu'il est vraiment homme et vraiment Dieu.

Grâce à cela, nous pouvons, avec Tavard, aller encore plus loin, car : «II y a Discours de Dieu adressé à l'homme parce que d'abord il y a Discours en Dieu, Discours de Dieu à Dieu. Dieu est le Locuteur, et aussi l'Auditeur, et encore le Discours $"{ }^{26}$ Cela veut dire que, avant tout, il y a communication et échange de parole à l'intérieur même de la Trinité et celle-ci manifeste par là son désir de s'extérioser dans le monde créé, de s'autocommuniquer. Cela fait, nous allons maintenant remonter encore une fois vers notre point de départ : «Au commencement était le Logos» (Jn 1, 1). Vers cette Parole de vie, toujours prononcée, en vue de mieux trancher le sens ontologique de cette Révélation, qui a rendu possible l'accueil du Verbe de Dieu en devenir pour nous et en chacun de nous.

\section{ii. "Au commencement était le Logos » $(J n$ 1, 1)}

Tout d'abord, ce mouvement vers le commencement nous sera possible parce que nous avons déjà franchi plusieurs seuils : premièrement, celui de la liberté de se décider pour ou contre la lumière («Ce qui fut en lui était la vie, et la vie était la lumière des hommes, et la lumière luit dans le ténèbres et les ténèbres ne l'ont pas saisie »- Jn 1, 4-5) ; ensuite, celui du refus de l'incrédulité par l'adhésion de foi («II est venu chez lui, et les siens ne l'ont pas accueilli. Mais à tous ceux qui l'ont accueilli, il a donné pouvoir de devenir enfants de Dieu, à ceux qui croient en son nom, eux qui ne furent engendrés ni du sang, ni d'un vouloir de chair, ni d'un vouloir d'homme, mais de Dieu »- Jn 1 , 11-13); pour, finalement, bénéficier de l'accomplissement et recevoir grâce sur grâce («Oui, de sa plénitude nous avons tous reçu, et grâce pour grâce »-Jn 1, 16).

Véritablement cette Parole est le commencement du temps et c'est bien en ce sens qu'elle est parfaitement identifiée en Logos de Dieu,

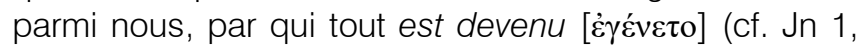
3). C'est pourquoi seulement à partir de l'esquisse d'une théologie discursive, nous pouvons proposer désormais un parcours vers la théologie du salut et de la rédemption. Un tel itinéraire s'origine dans l'enseignement de l'Écriture et reprend la Tradition, du fait que celle-ci souligne que la médiation de JésusChrist s'accomplit au cœur de deux mouvements croisés : celui qui va de Dieu vers nous dans le Christ

\footnotetext{
${ }^{26}$ Ibidem, p. 139
}

qui nous aime à en mourir ; et celui qui va de nous à Dieu par le Christ qui, dans son humanité, aime le Père à en mourir.

Dans cette perspective, la remontée aux origines éternelles des Personnes divines, dont nous avons déjà, d'une certaine manière, accompagné le devenir dans le monde et dans l'histoire, confirme et manifeste la nécessité d'entreprendre ce mouvement inverse. Car, au sein de cette dynamique et de ces allers-retours herméneutiques, nous apprenons que la naissance de Jésus, n'est pourtant pas un commencement absolu, mais la suite et l'accomplissement de la révélation du Logos divin, présent au monde depuis toujours, puis lié à l'histoire du peuple d'Israël, avant de s'enfoncer dans la temporalité de la chair du monde. ${ }^{27}$ En faisant cela, nous voulons mieux comprendre le mouvement du devenir du Logos éternel, lorsque nous évitons de placer l'Incarnation comme unique point de départ pour la christologie. Nous cherchons à apporter de nouvelles réflexions qui puissent éclairer davantage l'événement de cette venue de Dieu vers l'homme depuis le commencement du temps, en essayant de trouver un langage dans lequel nous pouvons penser en même temps la foi de l'Église.

Ainsi, notre option de mettre au tout début en évidence l'acte de lecture du Prologue de l'évangile selon saint Jean s'éclaire du fait qu'il est le seul texte du Nouveau Testament à prononcer les mots « devenu chair ». II situe par là l'Incarnation du Logos dans la continuité d'une histoire de révélation, dont le terme est le dévoilement de la vérité de Dieu par son Fils unique Jésus-Christ. C'est pourquoi cette première analyse a fait ressortir la simplicité absolue de Dieu, source d'une richesse inépuisable d'expression, un Discours absolument parlant et illimité.

En fait, si l'analyse du discours ne saurait ni compter ni mesurer les actualités infinies du Logos divin, ce ne serait pas là une excuse pour ne pas tenter de découvrir, dans les limites de l'analogie, quelles opérations syntactiques pouvaient correspondre aux caractéristiques du Logos divin. Car c'est dans les opérations d'une espèce de syntaxe théologique qu'il faut chercher une telle grammaire, du fait que cela présume sans doute que la théologie soit elle-même discours et qu'il existe un langage théologique. Cela veut dire, comme nous l'avons déjà vu, que le Prologue de l'évangile selon saint Jean, dès son verset initial, nous engage dans un acte discursif: "Au

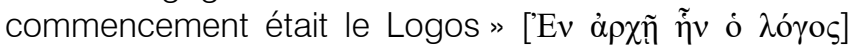
(Jn 1, 1).

À l'intérieur du Prologue, le Logos est à l'œuvre et il s'énonce toujours actuel. «Au commencement était le Logos ». II y demeure à jamais. Ce Logos exprime une

\footnotetext{
${ }^{27}$ Cf. MOINGT, J. « Dieu qui vient à l'homme, t. II-2 », p. 383.
} 
référence prioritaire au commencement. II habite l'ordre du commencement. D'ailleurs, le verbe était qui l'accompagne corrige l'idée de commencement comme un processus indéfini sur l'axe temporel délimité par un avant qui précède un après. "Sans verser dans le discours ontologique, il [le commencement] situe pourtant au plan de l'être, c'est-à-dire à celui d'un fondement qui neutralise les fluctuations et les aléas du temps et de l'histoire, au profit de l'instauration d'une permanence ${ }^{28}$

C'est ainsi que le temps verbal de l'imparfait, employé à plusieurs reprises dans le premier verset du quatrième évangile, prend la signification d'un passé qui influe sur le présent: "Au commencement était le Logos, et le Logos était auprès de Dieu, et le Logos était Dieu » (Jn 1, 1). Le Logos marque par là un ancrage ferme, solide, de telle sorte que la seule répétition du verbe être à l'imparfait tout au long du Prologue confère à cette structure narrative un Logos présent depuis le commencement, sous plusieurs formes, et qui y reste à jamais. Cette richesse sémantique rejaillit sur le Logos, en lien direct avec le verbe être, en le précisant et en le nuançant. "En Lui était la vie, et la vie était la lumière des hommes » (Jn 1, 4). "Il était la lumière véritable, qui illumine tout homme, venant dans le monde. II était dans

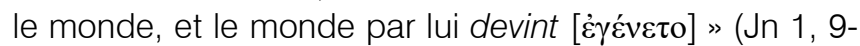
10).

C'est pourquoi, effectivement, oser penser maintenant à ce devenir de l'Être divin accordé au déploiement de la trinité économique dans le temps, exige faire la conciliation de l'éternité et du temps, une idée de Rahner, selon qui l'immuable en soi peut luimême être muable en l'autre. Cette idée nous met sur le chemin d'une solution possible pour essayer de répondre à notre question initiale, en vue d'intégrer l'événement de l'Incarnation à l'éternité de la vie divine. Cela confirme notre choix de ne pas prendre I'Incarnation immédiatement pour elle-même, "car un événement temporel ne peut avoir sa vérité dans l'éternité de Dieu qu'à condition de porter la «marque du définitif», mais cela est rendu vrai, « rétrospectivement », par le « détour » de la résurrection de Jésus ${ }^{29}$

Ayant donc posé ces prémisses, à la fois discursives et ontologiques, les mouvements de descente et de remontée prennent du sens. Car il fallait construire l'argumentation de ce devenir du Logos divin à partir de l'identité de Jésus en relation immédiate avec son Père (autre idée de Rahner), une fois que la personne est relation d'abandon de soi à l'autre (selon une définition de Hegel). En effet, Jésus a vécu son existence d'homme sous le mode de l'unité avec Dieu («Moi et le Père nous sommes un » - Jn 10, 30 ou «Qui m'a vu a vu le Père » - Jn 14, 9) à qui il s'est abandonné

\footnotetext{
${ }^{28}$ SIMOENS, Y. Selon Jean. 2. Une interprétation, p. 30

${ }^{29}$ MOINGT, J. « Dieu qui vient à l'homme, t. II-2 », p. 387
}

jusqu'à se livrer totalement à Lui par la mort ; et Dieu, en le ressuscitant, a ratifié la prétention de Jésus, a accepté le don qu'il lui a fait, l'a reçu en communion avec son être éternel et S'est révélé en tant que Père, de sorte que Jésus peut être légitimement identifié au Fils éternel connoté par ce nom.

Ainsi, à l'autre extrémité de la durée de la vie humaine de Jésus, sa mort et surtout sa résurrection atteste que lui, du fait de son union à Dieu, possède subsistance, depuis toujours, dans la personne du Fils. Cette subsistance il l'assume en la sauvegardant intégralement. "Le Verbe a donc créé l'humanité de Jésus en l'assumant, et l'a assumée en la créant ", selon une formule d'Augustin reprise par Rahner. ${ }^{30} \mathrm{C}$ 'est pourquoi nous osons dire qu'un individu n'est pas un commencement absolu, une création nouvelle, puisqu'il s'intègre et s'articule à la succession des moments du temps. Et Jésus lui-même est né dans une culture, en ayant une langue nationale propre, façonné par l'histoire des générations qui l'ont précédé.

Cela confirme donc notre choix de prendre un autre chemin, dans cette chaîne herméneutique $d u$ devenir, ce qui met en évidence à la fois la dynamique descendante et la dynamique ascendante, à partir de la signification de la résurrection de Jésus. Nous voulons montrer par là qu'il est forcément nécessaire, à la suite de l'Écriture et de la tradition de l'Église, d'analyser les diverses métaphores et catégories à travers lesquelles la réalité du salut se trouve exprimée dans la révélation et la foi. Ce mouvement du devenir, mis en évidence par la montée et la descente, va souligner que toutes ces catégories, malgré leur solidarité et leur complémentarité, resteront en elles-mêmes toujours plus pauvres que la personne de Jésus à partir de laquelle elles prennent sens. En d'autres termes, bien qu'elles puissent parler de révélation, de rédemption, de libération, de divinisation, de justification, du côté descendant ; ou de sacrifice, d'expiation-propitiation, de solidarité, voire de satisfaction, du côté ascendant, ces perspectives ne sont que des qualificatifs de la personne et de l'action de Jésus.

Au fond, le Christ se présente à nous comme médiation d'une Altérité qui sauve. Et justement en Jésus, mort et ressuscité, chaque être humain réalise non pas seulement que Dieu existe, mais qu'il veut être son libérateur et lui donner sa propre vie. II l'aime et l'homme existe et toute sa vie prend sens et valeur éternelle. L'homme découvre par là que l'acte sauveur que Dieu accomplit pour lui est aussi l'acte d'un homme comme lui, engageant librement sa vie dans une mission de salut, nous arrachant tous aux forces du mal et réalisant en Lui, pour nous, le passage à Dieu son Père. Dès lors, celui qui nous réconcilie avec Dieu et nous communique une telle adoption filiale est l'unique

\footnotetext{
${ }^{30}$ Cf. RAHNER, K. « Jésus Christ ». Dans : Traité fondamental de la foi, p. 252.
} 
Médiateur, parce qu'il est vraiment Dieu et vraiment homme.

Mais où avons-nous été entraînés ? Maintenant nous nous rapprochons de notre seconde question, ce qui va nous mener encore plus loin. Puisque celui qui est immuable en Lui-même nous fait signe qu'il peut être muable en un autre. Ce faisant, dit Rahner, nous aurons atteint un point extrême de l'ontologie : Dieu fait sienne la nature humaine. La nature indéfinissable assume l'être humain comme sa propre réalité. Dieu assume la nature humaine, parce que celle-ci est essentiellement une nature ouverte, assumable, parce qu'elle seule peut exister en une identité qui la dépasse elle-même et, seule, atteint par là la perfection même de son sens spécifique qui est incompréhensible.

Au plus profond l'homme n'a pas de choix: ou bien il se considére finalement comme un pur néant, que l'on inventorie pour constater, avec le rire cynique des damnés, qu'il n'y a rien dedans. Ou bien (puisqu'il n'est certainement pas lui-même la plénitude qui pourrait légitemement reposer en elle-même), il est visité par l'Infini et devient de cette façon celui qui ne s'invente pas lui-même, parce que la finitude humaine ne peut être dépassée qu'en s'enfonçant dans la plénitude incompréhensible de Dieu. ${ }^{31}$

«Et le Logos est devenu homme» - dit la phrase que nous désirons mieux comprendre. C'est justement en acceptant comme grâce insigne pour nous la charge de l'histoire et du devenir que nous allons confesser désormais un tel Dieu. Un Dieu trine qui se trouve, comme dit Joseph Moingt, sans muraille protectrice, au cœur d'un monde de possibles, que son désir d'aimer rend possible : un Dieu qui n'attend d'être aimé pour venir à l'être. L'explosion d'amour trinitaire fait donc que Dieu n'est pas seul en Lui-même, mais rend possible que l'autre soit et fait que l'autre n'est pas le seul à être. L'autocommunication de la Trinité à l'intérieur de soi est donc toujours en train de s'extérioriser.

\section{b) Le Devenir}

Fascinant mais difficile, le concept du "devenir " est de ceux qui échappent quand nous croyons les saisir. Toutefois, malgré ces difficultés, nous essayerons d'exposer ce que des philosophes comme Hegel, Gilles Deleuze et Félix Guattari pensent sous le nom de « devenir ", avant même d'opérer le passage au domaine théologique. Cet effort de lecture et ces contributions philosophiques visent à jeter un peu plus de lumière sur notre réflexion à propos du devenir en vue de parvenir à comprendre le devenir-homme du Fils de Dieu, chez Rahner.

Parvenus donc à ce point, bien que Rahner signale que la philosophie et la théologie sont encore en train de balbutier au sujet du « devenir ", il nous semble qu'un espace d'interlocution et de confluence peut s'ouvrir pour considérer sérieusement des deux côtés

\footnotetext{
${ }^{31}$ RAHNER, K. « Méditation 12 : L'Incarnation de Dieu », p. 169.
}

cette proposition «Et le Logos s'est fait chair » (Jn 1, 14), à savoir: d'une part, ce que dit la philosophie à propos du devenir et, d'autre part, comment se manifeste la théologie quand elle admet le devenir du Logos de Dieu, en même temps qu'elle confesse l'immutabilité de l'Être divin.

La foi chrétienne se trouve ainsi confrontée à un défi radical, étant donné que la philosophie grecque, surtout néoplatonicienne, s'était élevée à un sens aigu de la transcendance divine. Être vraiment chrétien exige donc de creuser au plus profond pour aller jusqu'au bout de ces énoncés quand nous confessons croire à l'Incarnation du Logos. Pour cela, en faisant l'exercice de changer l'axe de notre lecture de l'«Être» au "Devenir ", nous allons visiter une fois de plus ce que nous dit la philosophie à propos du devenir de l'être.

\section{i. Le devenir : "une synthèse disjonctive »}

Pour tenter de saisir le "devenir», nous proposons paradoxalement de relire ce que disait le philosophe grec présocratique Parménide : «l'être est et le non-être n'est pas". Alors, pour commencer, nous voyons déjà que l'affirmation de l'être et l'affirmation du néant sont équivalentes et qu'elles basculent l'une dans l'autre. C'est pourquoi nous allons oser dire que le dépassement de cette situation doit s'effectuer par le concept du devenir : l'incessant passage de l'un dans l'autre - de l'être au devenir.

De fait, quand nous passons à la lecture de la philosophie de Hegel, si nous disons «être » et que quelqu'un nous demande «être quoi ? » ou « quel être? ", nous devons répondre «être tout simplement » ou bien « être et rien de plus ». Car l'être pur est l'être sans aucune détermination. Ainsi, selon la perspective hegelienne, l'être n'est rien de plus sous peine de n'être plus l'être. D'autre part, l'être absolument indéterminé, c'est le néant et le néant n'est rien. Par conséquent, il l'est. Néanmoins, l'être et le néant ne sont pas identiques, bien qu'ils aient un élément commun qui est leur indétermination. Ils sont aussi indéterminés l'un que l'autre. À cet égard, si nous disons que l'être est, nous ne disons rien. II faut que nous nous engagions encore plus loin. Et l'unité des deux n'est pas un mélange, au contraire, c'est un constant passage de l'un à l'autre dans lequel le sens de l'être s'enrichit.

C'est pourquoi l'être devient. Cet événement du devenir de l'être est maintenant plus concret par rapport à l'abstraction du départ. Cette démarche consiste à exposer par là le réel du plus abstrait au plus concret, mais en revenant au point de départ à la fin. Dès lors, un nouveau concept est né : le devenir. Le devenir comme un passage, le devenir comme une certaine manière d'être, du fait qu'en parlant du devenir, nous continuons à parler de l'être. En fait, c'est la première des modalités concrètes de l'être. Et cette modalité de l'être qui consiste à devenir c'est l'existence. L'être en devenir, c'est donc exister. Et exister, ce n'est pas 
simplement être, c'est ne pas cesser de se déterminer au sein d'un devenir qui est une épreuve constante du non-être. L'existence, c'est ainsi l'être travaillé dialectiquement par le non-être, et en conséquence l'être en devenir. En ce sens, nous sommes passés de «l'être est » à «l'être existe » pour arriver à «l'être en devenir ». Effectivement, déterminer l'être comme existence est le résultat positif d'une dialectique antérieure interne à l'être pur. L'existence est ainsi la contradiction surmontée dans le devenir de l'être face au non-être.

Cela dit, il nous semble qu'il y a déjà quelques pistes pour admettre un possible devenir de l'Être divin. Dans le sillage de cette communication de Dieu, nous allons donc avancer maintenant avec Deleuze et Guattari. Ces philosophes français affirment que "devenir », c'est sans doute changer: ne plus nous comporter ni sentir les choses de la même manière, bien que sans doute nous ne changions pas d'identité. La mémoire demeure, chargée de tout ce que nous avons vécu et le corps vieillit sans métamorphose. En outre, il nous semble que "devenir " signifie aussi que les données les plus familières de la vie changent de sens, ou que nous n'entretenons plus les mêmes rapports avec les éléments coutumiers de notre existence.

Faut-il pour cela l'intrusion d'un dehors? Deleuze et Guattari assurent qu'il faut d'emblée que nous soyons en contact avec autre chose que nousmêmes, que quelque chose nous arrive. Le "devenir" implique ainsi un lieu de rencontre dans un sens large du terme. Nous ne devenons nous-mêmes autres qu'en rapport avec un autre objet ou bien plutôt quelqu'un d'autre. D'autre part, ce dehors s'entend ici en un sens absolu: il ne s'agit pas simplement de ce qui est à l'extérieur de nous, mais aussi de sentir autrement, d'entrer en contact avec un monde d'évaluations et des sentiments, auparavant inconnus, au point d'être lancé hors de nous-mêmes. II s'agit ainsi d'une série d'intensités, de mouvements, de précipitations, de suspens, de changements dans une différenciation intensive.

Nous affirmons par là un monde de possibilités de vie et de perspectives. Puisque, à vrai dire, toute rencontre a pour «objet » un être en devenir, non qu'il soit en train de changer, mais parce que ce que nous captons de lui ne relève pas de caractères identitaires stables. Les couples maudits, dit Deleuze, sont ceux qui postulent une homogénéité entre les personnes quand une rencontre ou une relation ne relève pas en réalité d'une mise en commun. ${ }^{32}$ Pourtant quelque chose circule allant de l'un à l'autre et les reliant, sans être du tout commun à l'un et à l'autre.

En effet, une rencontre est constituée de deux expériences distinctes, qui ne peuvent être mises en

${ }^{32}$ Cf. DELEUZE, G. Pourparlers. Paris : Minuit, 1990, p. 177. commun mais qui s'impliquent mutuellement, se présupposent réciproquement. Je suis objectivement en rapport avec l'autre, ayant objectivement capté quelque chose de lui et lui de moi. II y a donc un devenir commun aux deux, unissant indiscutablement des vécus différents. Ce qu'éprouve l'un est inséparable du rapport avec l'autre, mais ils ne se confondent point ; par conséquent, les affects, de part et d'autre différents, ne se produisent pas l'un sans l'autre. Tentons, avec le philosophe François Zourabichvili, une première synthèse approximative :

Les termes de la relation se caractérisent par leur hétérogénéité radicale et irréductible [...] ; cette hétérogénéité n'empêche pas l'effectivité d'une relation qui, dès lors, se dédouble en deux rapports inverses mais solidaires, au lieu d'une relation simple entre un terme et un autre (la relation de l'un à l'autre n'est pas la même que celle de l'autre avec l'un); enfin (nous partions de là), cette double relation modifie l'économie interne de chacun des termes, et c'est pourquoi elle reçoit le nom de devenir [...]. La relation s'établit moins entre un terme et un autre qu'entre chaque terme et ce qu'il capte de l'autre, ou - cela est équivalent - entre chaque terme et ce qu'il devient, à la rencontre de l'autre. ${ }^{33}$

Désormais nous percevons mieux le risque d'une simplification et nous nous rendons compte combien les idées de fusion ruineraient le concept que Deleuze et Guattari mettent en évidence. Or, le "devenir" implique que quelque chose de l'autre, objectivement, se passe en nous. Le centre de la question, dit Zourabichvili, est celui d'une identification sans identité, d'une communication sans mise en commun, d'un rapport qui ne supprime pas l'hétérogénéité des deux termes, d'une relation dont nous affirmons jusqu'au bout à la fois l'effectivité et l'extériorité. En fait, le problème est de penser jusqu'au bout une relation à l'autre en tant qu'autre - insistent Deleuze et Guattari -, opérant par la différence et non par la similitude. II y a rencontre où chacun pousse l'autre, l'entraîne dans sa ligne de fuite. Ce n'est pas affaire d'imitation, mais de conjonction.

En fait, nous parlons aussi de sympathie pour autant que capter, envelopper les rapports de l'autre, revient à envelopper la manière de sentir de l'autre. Capter la manière de sentir et de se rapprocher fait que nous soyons capables de laisser une sensibilité étrangère travailler la nôtre, agir en elle au point de la changer. D'une certaine façon, cette sensibilité autre sentira comme nous sentons; de même, notre vie et notre affectivité l'envelopperont. L'intérêt de Deleuze et Guattari consiste vraiment à penser cette rencontre, ou l'affect au sens fort du terme. Autrement dit, penser l'affect de la rencontre ou bien la résonance de l'un dans l'autre. Or si un autre sentir s'est objectivement logé en nous, bien qu'il ne soit pas le nôtre, et qui

33 ZOURABICHVILI, F. "Qu'est-ce qu'un devenir, pour Gilles Deleuze ? » (Conférence prononcée à Horlieu, Lyon, le 27 mars 1997). 
pourtant lui est attribuable, envelopper une autre sensibilité veut dire que nous sentons d'une façon autre que la nôtre.

Du point de vue philosophique, selon Deleuze et Guattari, envelopper l'autre dans une rencontre signifie incorporer à ses propres rapports des rapports hétérogènes comme hétérogènes. Un autre sentir se trouve objectivement en nous, de telle sorte qu'envelopper une autre sensibilité fait surgir un autre que nous. Cela veut dire en effet que nous sentons d'une façon autre que la nôtre. Objectivement cette rencontre nous fait sentir autrement, nous fait gagner une zone de nous-mêmes, nous fait sentir nous-mêmes autrement. C'est bien là l'irréversibilité du devenir. Deleuze et Guattari signalent donc par le devenir philosophique une synthèse de l'hétérogène: un hétérogène en enveloppe un autre; un hétérogène résonne dans un autre.

En conséquence, "une synthèse disjonctive » prend la place et intervient à cet endroit. C'est-à-dire : les deux individus déplacent leur singularité, ils jalonnent un parcours d'existence, l'un se sent affecté par l'autre par cet «événement du devenir » où l'affect est inséparable de l'expérience d'une possibilité de vie. L'un ne devient que par son rapport à l'autre. Dans cette "synthèse disjonctive", tous deux se sont donc épousés, ils conjuguent leurs forces et quoique " distincts » au niveau de leur expression et contenu, ils deviennent indiscernables. En revanche, leur identité n'efface point la différence des natures. Voilà une réunion d'identités sans équivalence, une vraie communication : un événement se déroulant sur deux plans différents.

\section{ii. «Et le Logos s'est fait homme » (Jn 1, 14)}

Si du côté philosophique une "synthèse disjonctive " irréversible s'annonce quand nous accompagnons les mouvements de l'être en « devenir », comme nous venons de le voir, du côté théologique, il nous semble que nous nous rapprochons du devenirhomme du Fils de Dieu. Mais pour réaliser cela, Rahner affirme qu'il faudrait que le philosophe et le théologien abandonnent complètement le champ qui est le leur, et que, usant d'une méthode qui relève davantage de l'a posteriori, la méthode des sciences de la nature, ils déploient les structures fondamentales de I'histoire du monde. Selon lui, il est souhaitable que nous puissions montrer d'une façon plus concrète quels traits communs existent dans le devenir de ce qui est matériel, de ce qui est vivant, et de ce qui est spirituel. Plus précisément, comment ce qui est simplement matériel prélude, dans sa dimension propre, à la réalité supérieure de la vie, en une approche progressive de la frontière qui, par le jeu de l'autotranscendance, est à déborder en direction de l'esprit.

Rahner commence donc par admettre que si le monde est un et si, comme un, il a une histoire en devenir, tout n'est pas là dès toujours et d'emblée. II n'y a donc aucune raison de nier que la matière avait à évoluer en direction de la vie et de l'homme. Ce que de la sorte nous saisissons comme conceptuellement pensable: les sciences de la nature conçoivent un monde en devenir, dans lequel l'homme entre en scène comme produit de ce monde. Cette histoire est donc vue comme une histoire cohérente de la matière, de la vie et de l'homme. Cette histoire une n'exclut pas des différences essentielles, mais elle les inclut. Ainsi, l'histoire n'est pas la permanence du même, mais le devenir de ce qui est toujours nouveau, de ce qui est plus, et pas seulement de ce qui est autre, comme disent les philosophes. De ce fait, cette histoire s'affirme justement dans une autotranscendance essentielle.

Dans la mesure donc où l'ordre supérieur englobe toujours l'ordre inférieur comme persistant en lui, il est clair que ce qui est inférieur, dans le développement de sa réalité et de son ordre propres prélude à l'avènement proprement dit de l'autotranscendance en ce qu'il la prépare; il se met lentement en mouvement vers ces limites, dans une histoire qui ensuite se déborde elle-même dans l'autotranscendance proprement dite; vers ces limites que l'on reconnaît clairement dépassées seulement à partir d'un déploiement plus clair de ce qui est nouveau, sans qu'on puisse les fixer en elles-mêmes avec précision et clarté. ${ }^{34}$

En ce sens, pour Rahner, si l'homme est l'autotranscendance de la matière vivante, l'histoire de la nature et l'histoire de l'esprit forment en l'homme une unité intérieure. Par conséquent, l'histoire de la nature évolue vers l'homme, se poursuit en lui comme son histoire personnelle et en lui est conservée et dépassée. De telle sorte que l'histoire de la nature humaine parvient à son but propre avec l'histoire de l'esprit et en elle. Autrement dit, l'histoire de la nature vient à son but dans l'histoire libre de l'esprit, et demeure là comme son constitutif intérieur. Ainsi, dans la mesure où l'histoire de l'homme inclut toujours en elle l'histoire de la nature, elle se trouve toujours au sein de sa liberté. Cela dit, l'homme n'est pas forcément un observateur spirituel de la nature, mais il en est partie prenante, parce qu'il doit mener plus avant son histoire. Et son histoire n'est pas simplement une histoire culturelle superposée à l'histoire de la nature, mais elle est aussi et surtout une transformation active de ce monde.

C'est ainsi seulement par une action spirituelle, et par conséquent par une spiritualité qui est action, que l'homme et la nature viennent de concert à leur but unique et commun. L'histoire de la liberté de l'esprit est donc enveloppée par la grâce de Dieu. De ce fait, le chrétien sait que l'histoire du cosmos, en tant que totalité, trouve son accomplissement réel malgré la liberté de l'homme, en elle et par elle. De là, l'essence

\footnotetext{
${ }^{34}$ RAHNER, K. « Jésus Christ ». Dans : Traité fondamental de la foi, p. 214.
} 
de l'homme peut être vue à l'intérieur d'une conception fondamentale et globale du monde.

« [...] c'est justement cette essence de l'homme qui, par son autotranscendance suprême, libre, rendue gratuitement possible et pleine pour lui par Dieu même, cette autotranscendance qui, par l'autocommunication de Dieu, le porte jusqu'en Dieu même, qui «attend" son accomplissement et celui du monde dans ce que, en concepts chrétiens, nous appelons grâce et gloire ${ }^{35}$

Rahner considère aussi que le point de départ permanent et la garantie absolue en ce que cette autotranscendance, fondamentalement insurpassable et irréversible, réussit et se trouve déjà engagée, c'est ce que nous appelons « l'union hypostatique ». En ce sens, pour lui, I'Homme-Dieu est l'origine première de la réussite définitive du mouvement d'autotranscendance du monde qui le porte vers la proximité absolue du mystère de Dieu. Cette union hypostatique ne saurait être vue comme quelque chose qui différencie Jésus de nous, mais comme quelque chose qui doit advenir une fois et une fois seulement alors que le monde commence d'aborder sa phase dernière, son point culminant définitif et sa proximité radicale du Mystère absolu qui est Dieu Lui-même.

De là l'Incarnation apparaît comme l'origine nécessaire et permanente de la divinisation du monde en son ensemble. Dans la mesure où advient, en ouverture sans réserve, la proximité insurpassable au mystère absolu que Dieu est et demeure, et dans la mesure où cette phase définitive de l'histoire du monde certes déjà commencée, mais n'est pas encore achevée, le cours ultérieur de cette phase et son résultat demeurent naturellement enveloppés de mystère. ${ }^{36}$

Ce qui est clair et définitif dans la vérité chrétienne, c'est la remise inexorable de l'homme au Mystère. D'autre part, ce qui devient de plus en plus évident, selon Teilhard de Chardin, dans le cadre d'une évolution cosmique, c'est la loi de complexitéconscience. L'homme n'est pas un composé contradictoire, mais une unité (esprit et matière). Cette relation de réciprocité n'est pas simplement une relation statique, mais elle aune histoire. II faut pour cela comprendre l'esprit et la matière, sans les séparer, comme des moments de l'homme en son unité, moments rapportés l'un à l'autre, inséparables et irréductibles.

C'est pourquoi il faut appliquer à la multitude humaine la loi fondamentale de l'évolution. Pour cela, Teilhard de Chardin explique que, de même que les cellules vivantes sont irrésistiblement poussées à s'unir entre elles, en arrangements de plus en plus complexes, de même les hommes sont irrésistiblement poussés à organiser et perfectionner leurs rapports sociaux. D'après lui, il existe une nécessité biologique en route. L'humanité marche donc vers des formes de

\footnotetext{
35 Ibidem, p. 208.

${ }^{36}$ Ibidem, pp. 208-209.
}

socialisation de plus en plus étendues, de plus en plus complexes et de plus en plus interdépendantes. II serait donc inexplicable que cette évolution ne s'achève pas en un Dieu personnel, en un centre de convergence qu'il appelle le point Oméga et qui s'identifie à Jésus de Nazareth, devenu le Christ universel, tel que l'ont annoncé les écrits de saint Paul et de saint Jean.

Cela dit, Rahner nous invite encore une fois à considérer en toute simplicité que Dieu peut devenir quelque chose. II insiste que Celui qui est immuable en Lui-même peut être muable en un autre, car Celui qui est l'Absolu a, dans la pure liberté de son indépendance infinie, qu'll conserve toujours, la possibilité de devenir l'autre, de devenir-homme. II est donc possible que Dieu, précisément en se dépouillant, se dessaisissant de soi, fasse de l'homme sa propre réalité. Et selon la foi, d'après lui :

Le phénomène par excellence est bien plutôt et tout justement le dépouillement de soi, le devenir, la kénôsis et la génésis de Dieu lui-même, qui peut devenir, parce qu'en faisant jaillir de soi quelque chose d'autre, il devient luimême ce qui a jailli, sans pour autant lui-même devenir ce qui lui est propre, qui est l'origine même de tout. Parce que tout en demeurant dans son infinie plénitude, Dieu se dessaisit de lui-même (parce qu'il est l'Amour, c'est-à-dire la volonté de combler le vide - qui possède de quoi combler), l'autre prend consistance comme sa propre réalité à lui, en ce qu'il le possède comme sien. Inversement: parce qu'il veut vraiment posséder l'autre comme son bien propre, il l'établit dans sa réalité authentique. Dieu sort de lui-même, en personne, comme la plénitude qui se donne. ${ }^{37}$

Rahner fait remarquer par là que Dieu agit ainsi parce que, primordialement, il peut (et non il doit) librement s'introduire dans l'histoire, que l'Écriture l'appelle l'Amour, l'Amour dont la liberté prodigue est tout simplement indéfinissable. Par conséquent, dans la capacité qu'll a de créer, de faire exister, de faire sortir le tout autre en soi de son propre néant, sans cesser pour autant d'être Lui-même, il n'est qu'un pouvoir dérivé, limité, secondaire, qui se fonde en définitive sur ce pouvoir primordial, bien que le pouvoir de créer soit dissociable de celui d'aimer. En effet, dans ces mouvements, le Créateur exprime son désir libre à la fois de se communiquer en acte d'Amour dans la Création tout entière et de s'autocommuniquer par I'Incarnation de son Logos divin.

Dès lors, nous parviendrons à mieux comprendre que c'est précisément le Logos divin qui est devenu homme, et qui est le seul à pouvoir le devenir, car la Parole qui fait advenir l'univers ne s'adresse pas à des choses qui ne sont pas susceptibles d'y répondre. Cette Parole exprime d'ailleurs une communication d'un sujet à l'autre et, d'une certaine façon, c'est un discours qui s'énonce, comme nous l'avons vu. La Parole n'est pas sans la

${ }^{37}$ RAHNER, K. « Méditation 12 : L'Incarnation de Dieu », p. 173. 
réponse qu'elle appelle et suscite - comme nous le montre Joseph Moingt :

La parole créatrice est celle que Dieu adresse à un être capable de lui répondre, qu'll crée pour cela capable de parler et dont la parole est associée immédiatement à celle de Dieu : unique Parole créatrice qui sépare du cosmos un sujet parlant et qui se diffracte dans la multiplicité des noms que celui-ci, en réponse à Dieu, donne aux choses pour les séparer de l'informe. II fut ainsi fait, par un dire unique et commun, parce que Dieu n'est pas sans l'homme (comme l'a dit justement Karl Barth), ni l'homme sans le monde (ce qu'il a peut-être moins bien vu). [...] voilà pourquoi le chrétien interprète la parole créatrice comme celle qui s'échange au sein de la Trinité entre le Père et son Verbe dans le « milieu » communiquant qui est l'Esprit Saint. ${ }^{38}$

De fait, la manière dont nous avons compris le "dire créateur", en tant que dispositif énonciatif au cœur du Prologue, commande "l'intelligence du faire » et de son "devenir dans le monde». Si ce dire est conçu dans l'ordre de la communication, de l'échange et de l'appel, le faire créateur et son devenir dans la chair de Jésus de Nazareth se laisseront comprendre dans le registre symbolique du don, de l'amour, d'une offre de liberté, d'une mise en relation, d'une histoire commençante, d'un partenariat, toutes significations qui sont les harmoniques d'une parole vive, du Logos éternel de Dieu Lui-même.

De ce fait, Joseph Moingt souligne que la parole créatrice ne saurait être autre que celle par laquelle Dieu se dit Lui-même par son Logos. Car il n'y a qu'une Parole, et l'acte de parler n'est rien d'autre pour Lui que l'acte d'être, à savoir : d'exister en relation à un autre. Ainsi, Dieu est Père par l'acte de se dire dans et à un autre, qui existe comme un autre lui-même. Or, l'Être de Dieu, soustrait à toute nécessité comme à toute détermination, ne se définit par rien d'autre que par la liberté d'être Soi-même. C'est ainsi que le Logos reçoit la parole que lui adresse le Père comme l'appel à exister dans la liberté d'un être-autre, comme l'appel de son amour infini à se répandre dans le monde. II exprime donc au Père son consentement, et cet accord réciproque, qui scelle la relation du Père au Logos dans leur être commun, donne l'existence à l'Esprit Saint, lien vivant de leur intercommunication, preuve en acte que le Père reste uni au Logos en son être-autre, quand il s'énonce dans le monde.

En outre, bien que Rahner nous ait fait remarquer qu'il ne s'agissait pas de prouver le sens de ce devenir-homme par les déclarations du Magistère officiel de l'Église, il admet pour autant que quiconque se tourne vers une véritable compréhension du Mystère doit, dans un mouvement spirituel, prendre distance à l'égard des formules dogmatiques et, de retour à elles, trouver le sens de ce qu'il a compris dans son parcours existentiel et herméneutique. À cet effet, les énoncés de

\footnotetext{
${ }^{38}$ MOINGT, J. « Dieu qui vient à l'homme, t. II-2 », p. 293
}

La Constitution dogmatique sur La Révélation Divine (Dei Verbum) s'enrichissent et prennent tout leur sens quand ils affirment qu'en ces jours qui sont les derniers, Dieu nous a parlé par son Fils (cf. He 1, 1-2), après avoir, à bien des reprises et de bien des manières, parlé par les prophètes. De même, quand ils annoncent qu'll nous a envoyé son Fils, le Logos éternel qui éclaire tous les hommes, pour qu'il demeure parmi nous et nous fasse connaître les profondeurs de l'Être divin en devenir (cf. Jn 1, 1-18).

"Jésus-Christ donc, le Verbe fait chair, "homme envoyé aux hommes ", "prononce les paroles de Dieu » $(\mathrm{Jn} 3,34)$ et achève l'œuvre de salut que le Père lui a donnée à faire (cf. Jn 5, $36 ; 17$, 4). C'est donc lui - le voir, c'est voir le Père (cf. Jn 14, 9) - qui, par toute sa présence et par la manifestation qu'il fait de lui-même par ses paroles et ses œuvres, par ses signes et ses miracles, et plus particulièrement par sa mort et sa résurrection glorieuse d'entre les morts, par l'envoi enfin de l'Esprit de vérité, achève en l'accomplissant la révélation, et la confirme encore en attestant divinement que Dieu lui-même est avec nous pour nous arracher aux ténèbres du péché et de la mort et nous ressusciter pour la vie éternelle. L'économie chrétienne, étant Alliance Nouvelle et définitive, ne passera donc jamais et aucune nouvelle révélation publique n'est dès lors à attendre avant la manifestation glorieuse de notre Seigneur Jésus Christ (cf. 1 Tm 6, 14 ; Tt 2, 13). ${ }^{39}$

Désormais, nous comprenons mieux ce que veut dire : le devenir-homme du Fils de Dieu. L'ouverture au Mystère et à sa dynamique propre sont la condition de possibilité d'une saisie compréhensive de ce Tout singulier et originaire en devenir en nous et pour nous. Dès lors, c'est Jésus-Christ lui-même qui nous invite à une compréhension beaucoup plus radicale de notre vie humaine, car «[...] le Logos devient homme, son humanité n'est pas donnée à l'avance; elle est ce qui devient et naît à l'essence et à l'existence dans la mesure exacte où le Logos de fait se dépouille de soi $\gg{ }^{40}$ Cet homme est précisément comme homme dessaisissement de Soi de Dieu dans leur dessaisissement de soi commun. Car, pour Rahner, Dieu saisit dans sa révélation justement quand II se dessaisit de Lui-même, quand II se révèle comme l'Amour, quand II voile la majesté de cet amour et se montre sous les traits ordinaires de l'homme. Effectivement, II est l'Amour et, par nécessité de nature, la merveilleuse possibilité de Se donner librement.

En conséquence, Rahner nous donne la possibilité d'ébaucher une définition de l'homme, le prédicat de cette proposition initiale : «Et le Logos s'est fait homme » (Jn 1, 14), en pénétrant dans l'abîme de ce que son mystère a de plus profond et de plus obscur. Ce qui naît de la liberté de Dieu, quand Celui-ci se dit Lui-même et profère sa Parole dans le vide du néant.

\footnotetext{
39 DOCUMENTS DE VATICAN ॥ (Constitution Dogmatique sur la Révélation Divine) : DV 4.

${ }^{40}$ RAHNER, K. « Méditation 12 : L'Incarnation de Dieu », p. 174.
} 
Rahner appelle le Logos incarné le chiffre de Dieu et, selon lui, ce chiffre est l'homme, plus précisément, le Fils de l'homme, du fait que quand Dieu veut être nonDieu, l'homme apparaît.

Cela dit, si Dieu Lui-même est homme et le demeure dans l'éternité, aucune théologie n'a le droit de se faire une petite idée de l'homme. Car ce serait se faire une petite idée de Dieu, lequel demeure le Mystère sans fond - admet Rahner. L'homme est ainsi, dans l'éternité, le mystère proféré par Dieu, participant toujours au Mystère qui le fonde. En tant qu'il est mystère inépuisable dans la béatitude de l'amour, cet homme doit toujours être reconnu dans cette dignité : il est l'auto-expression de Dieu et, en même temps, l'être fini habité par la Parole infinie de Dieu.

Ainsi, de «l'Être » au «Devenir » du Logos divin, de même que pour tout être humain, nous nous trouvons dans un carrefour où la christologie est le terme et le commencement d'une anthropologie. D'abord parce que cette anthropologie, dit Rahner, Dieu Lui-même l'a formulée en proférant sa Parole comme notre chair dans le vide du non-Dieu et du péché. En ce sens, comme nous l'avons vu, cette théologie est devenu théologie discursive parce que nous ne pouvons pas la proférer sans passer par le Logos, le Verbe incarné dans la chair de Jésus de Nazareth, c'està-dire la chair de l'être humain tout simplement. Ce parcours et cette énonciation discursive du «Logos en devenir » nous obligent ainsi à confesser - par le Christ, avec lui et en lui - un Dieu qui est là où nous sommes et encore plus : que nous ne pouvons le trouver que là. En admettant cela, nous admettons aussi que :

S'il y demeure l'Infini, ce n'est pas à dire qu'll est infini «aussi » là et quelque part ailleurs, mais que le fini a acquis une profondeur infinie. II n'y a donc plus d'opposition à I'Infini, il n'y a plus que ce que l'Infini Lui-même est devenu, pour ouvrir à toute la finitude, au sein duquel il s'est Luimême fait « partie », accès à l'Infini ». ${ }^{41}$

En d'autres termes, pour les hommes, l'Infini s'est fait accès à I'Infini, dans l'exacte mesure où il est devenu la réalité du rien, où il est devenu homme. Cela entraîne des conséquences encore plus fortes et plus profondes pour tous les chrétiens. Car celui qui assume son être d'homme et confesse le Logos devenu homme dans la chair de Jésus de Nazareth, celui-là assume le Fils de l'homme, parce qu'en lui Dieu a assumé l'homme. Le devenir-homme du Fils de Dieu entraîne ainsi le devenir homme de chacun de nous, du fait que Dieu est devenu homme, c'est-à-dire: II est devenu notre prochain.

C'est là toute la Loi et les prophètes: «Tu aimeras le Seigneur ton Dieu de tout ton cœur, de toute ton âme et de tout ton esprit: voilà le plus grand commandement. Le second lui est semblable: Tu aimeras ton prochain comme toi-même » (Mt 22, 37-39).

${ }^{41}$ Ibidem, p. 176.
C'est là aussi la perfection dans l'amour : "Quant à nous, aimons, puisque lui nous a aimés le premier. Si quelqu'un dit: 'J'aime Dieu' et qu'il déteste son frère, c'est un menteur: celui qui n'aime pas son frère, qu'il voit, ne saurait aimer le Dieu qu'il ne voit pas » (1 Jn 4, 19-20). Grâce à ce commandement que nous avons reçu du Christ, "que celui qui aime Dieu aime aussi son frère » $(1 \mathrm{Jn} 4,21)$, nous passons maintenant au devenir homme de l'homme.

\section{c) Le devenir homme de l'homme}

Le parcours de «l'Être » au «Devenir », et plus précisément «l'énonciation du Logos en devenir ", au sein du Prologue de l'évangile selon saint Jean, nous a rendu capables de voir encore plus loin pour professer avec l'Église notre foi. Or, il a plu à Dieu, dans sa bonté et sa sagesse, de se révéler Lui-même et de faire connaître le mystère de sa volonté, dès avant la fondation du monde (cf. Ep 1, 3s). II détermina ainsi d'avance que nous serions pour Lui des fils adoptifs par le Christ, le Logos fait chair, en ayant dans l'Esprit Saint accès auprès de Lui, pour devenir participants de la nature divine. Dieu qui crée (cf. Jn 1, 3) et conserve toutes choses par le Verbe, donne ainsi aux hommes dans les choses créées un témoignage incessant sur Lui-même (cf. Rm 1, 19-20) ; voulant de plus ouvrir la voie du salut d'en haut, II s'est manifesté aussi Luimême, dès l'origine, à nos premiers parents. ${ }^{42}$

L'homme est donc un mystère. Non, il est le mystère ! - dit Rahner. Mais nous ne le sommes pas en étant indigence qui s'ouvre au Mystère de la plénitude incompréhensible de Dieu; au contraire, nous le sommes bien plutôt parce que Dieu exprime un jour ce mystère comme étant le sien. En ce sens, la créature humaine, à partir de son fondement essentiel le plus intérieur, est entendue comme possibilité de pouvoirêtre-assumée, du fait de fournir un «matériau » pour une possible histoire de Dieu. Ainsi, dans son devenirhomme, le Logos crée la réalité humaine en l'assumant, affirme Rahner, et l'assume en s'extériorisant lui-même. De fait, le Christ est le plus radicalement homme, et son humanité la plus autonome, la plus libre, parce qu'elle est l'humanité assumée, posée comme autodiction de Dieu. "C'est de façon créatrice que Dieu ébauche la créature en l'instituant à partir du néant, dans sa propre réalité, distincte de Dieu, comme la grammaire d'un auto-énoncé possible de Dieu ». ${ }^{43}$

La Révélation n'est donc pas seulement l'enseignement de Jésus dans les évangiles. Certes, en sa propre personne, il est le révélateur de Dieu et de par son humanité et sa cohérence interne il nous aide à devenir hommes à notre tour. Pour cette raison justement, il nous faut poursuivre l'annonce kérygmatique de cette Révélation aujourd'hui encore et

${ }^{42}$ Cf. DV 2-3.

${ }^{43}$ RAHNER, K. « Jésus Christ ». Dans : Traité fondamental de la foi, p. 252. 
susciter l'adhésion au Christ pour que d'autres puissent devenir hommes à leur tour, selon un processus d'identification mutuelle établie entre Jésus et ceux et celles qui croisent son chemin. Tout cela exprime bien les soucis de l'Apôtre: "Comment l'invoquer sans d'abord croire en lui ? Et comment croire sans d'abord l'entendre? Et comment l'entendre sans quelqu'un qui proclame? Et comment proclamer sans être d'abord envoyé ? " (Rm 10, 14-15). Car l'adhésion présuppose la foi, la foi présuppose l'écoute, l'écoute présuppose l'annonce et l'annonce de la parole a besoin que quelqu'un la porte et qu'il soit envoyé pour la répandre dans le monde. II faut donc élargir « le cercle du devenir homme» par l'énonciation du Logos divin, puisqu'il nous unit au Père qui est en lui et il nous fait communier à l'Esprit Saint pour devenir véritablement des êtres humains à son Image.

Jésus se présente ainsi comme l'homme pour et avec les autres. II se présente comme le signe même de l'Amour aux yeux de l'humanité, ce qui est à la racine de lui-même: I'homme pour un Autre. À vrai dire, cette histoire se réalise toujours et partout par l'offre de la grâce aux hommes libres de tous les temps et de toutes les conditions. Ainsi, la grâce de Dieu universellement à l'œuvre, comme le dit Rahner, présente une tangibilité historique lorsque, en certains points déterminés de l'espace et du temps, et du fait de leur enchaînement, Dieu, par la révélation de sa Parole atteste sa volonté de sauver le monde. De même, la Révélation nous donne accès au salut, du fait qu'elle anticipe la vision de Dieu, de telle sorte qu'elle est l'acte par lequel Dieu Se communique en communauté de vie tel qu'll est comme l'affirme Joseph Moingt. ${ }^{44}$ Et parce que la Révélation est l'œuvre du "Logos en devenir », depuis le commencement, il s'ensuit qu'elle est l'histoire de la Trinité pour nous, en nous et avec nous.

En outre, le Prologue johannique confère une portée universelle et éthique à notre histoire. II marque la continuité de l'histoire de la Révélation par la présence du Logos au commencement depuis la Création. II montre aussi la réalisation pleine de cette histoire par la venue du Logos dans la chair, par laquelle Dieu manifeste la plénitude de son Être pour nous, puisqu'll nous appelle à nous reconnaître en frères dans son Fils. De fait, "à tous ceux qui l'ont accueilli, il a donné pouvoir de devenir enfants de Dieu, à ceux qui croient en son nom, eux qui ne furent engendrés ni du sang, ni d'un vouloir de chair, ni d'un vouloir d'homme, mais de Dieu » (Jn 1, 11-13); pour, finalement, bénéficier de l'accomplissement et recevoir grâce sur grâce « Oui, de sa plénitude nous avons tous reçu, et grâce pour grâce » (Jn 1, 16).

C'est ainsi que l'idée du salut apparait depuis toujours comme constitutive de l'homme, puisque, selon Rahner, elle implique l'autocommunication de

${ }^{44}$ Cf. Cf. MOINGT, J. « Dieu qui vient à I'homme, t. II-2 », p. 335.
Dieu en Lui-même comme la force la plus intérieure de notre existence et comme notre but. Elle manifeste aussi notre lutte et notre aspiration à tout faire pour devenir ce que nous pouvons être et ce que nous désirons devenir. De là, si l'être humain se sent limité dans son accomplissement par la mort, par le mal et par la fatalité, pour sauver sa vie et la réussir, il doit surmonter tout ce qui y fait obstacle.

Néanmoins, le panorama chrétien du salut est bien plus large qu'il n'apparaissait, il touche à tout ce qui fait échec à la réalisation pleine de l'homme et il ne se limite pas au péché. Car, en effet, pécheur ou non, selon Bernard Sesboüé, ${ }^{45}$ l'homme est en toute hypothèse devant Dieu dans la situation d'un besoin radical de salut. En tant que créature, il est un être fini, habité par une vocation, celle de connaître Dieu, de le voir et de communier à sa propre vie. II ne peut pas s'accomplir parfaitement comme homme par ses propres forces. II ne peut pas à lui seul réaliser sa vocation, il ne peut s'accomplir comme homme qu'en Dieu.

D'autre part, cette idée de tenir d'un autre son accomplissement est funeste à l'homme, précisément parce qu'elle l'empêche de devenir lui-même par luimême. Ainsi, bien que nous comprenions cette objection et que Dieu soit devenu objet de soupçon pour tant d'hommes d'aujourd'hui, des théologiens comme Gesché soulignent qu'il faut y regarder de plus près et se demander s'il ne s'y trouve pas quelque malentendu qui touche à la méconnaissance de l'importance de l'altérité. ${ }^{46}$ Car l'homme n'est pas un être qui peut se passer des autres, et enfermé en luimême il perd son être. L'altérité est donc un facteur constitutif de l'identité humaine. L'autre est justement celui qui, par son altérité même, nous appelle, nous convoque, nous fait sortir de l'enfermement en nousmêmes et nous permet d'accéder au plus intime de nous-mêmes. L'autre devient à la fois pour nous : grâce et salut. En conséquence, toute autonomie commence par être provoquée par une altérité qui nous appelle à devenir pleinement les hommes que nous sommes.

En ce sens, le Christ se présente à nous comme cette médiation d'une altérité qui sauve. En Jésus, mort et ressuscité, chaque être humain réalise non pas seulement que Dieu existe, mais qu'll existe pour lui, avec lui et en lui et qu'll veut être son libérateur et lui donner sa propre vie. II l'aime et l'homme existe et toute sa vie prend sens et valeur éternelle. L'homme découvre ainsi que l'acte sauveur que Dieu accomplit pour lui est aussi l'acte d'un homme comme lui, engageant librement sa vie dans une mission de salut,

${ }^{45}$ Cf. SESBOÜÉ, Bernard. "Le sauver et le salut. Problématique». Dans : Jésus-Christ l'unique Médiateur. Essai sur la rédemption et le salut. Paris : Desclée, 1988, pp. 1-120.

${ }^{46}$ GESCHÉ, Adolphe. "Topiques de la question du salut ». Dans Dieu pour penser V. La destinée. Paris : Cerf, 1995, pp. 27-70. 
nous arrachant tous aux forces du mal et réalisant en lui, pour nous, le passage à Dieu son Père. Par ailleurs, celui qui nous réconcilie avec Dieu et nous communique une telle adoption filiale est l'unique Médiateur (cf. 1Tm 2, 5-6), parce qu'il est vraiment Dieu et vraiment homme.

De ce fait, Dieu Lui-même, dans sa grandeur inexprimable, dans son existence éternelle, nous a saisis et introduits dans cette existence éternelle qui lui appartient. Ainsi, Jésus-Christ, en tant qu'homme-Dieu, est à son tour la cause opérante, propre et unique, de notre salut. II est, en tant que Fils de Dieu, notre salut même et l'accès de grâce à Dieu le Père - soutient Rahner. D'autre part, en tant que celui qui, dans l'unité de sa personne, possède l'essence divine, venant du Père par génération éternelle, et la nature humaine, venant de la Vierge Marie, il est celui qui, dans son être et dans son œuvre, constitue l'unique et exceptionnelle réunion entre Dieu et sa création à racheter. II est en ce sens un Médiateur tel qu'il n'y en a pas d'autre que lui. De telle sorte que si tout homme veut réussir sa vie, l'idée du salut doit d'abord y faire écho.

Après Jésus-Christ, on ne peut rien dire de vrai, d'authentique, de concret sur Dieu sans le confesser comme Emmanuel, comme Dieu-avec-nous, comme le Dieu de notre chair, le Dieu de notre nature humaine, le Dieu de nos signes humains dans les sacrements, le Dieu de nos autels, comme le Dieu qui est né de la Vierge Marie et se trouve ainsi comme homme parmi nous, homme et Dieu en une seule personne. ${ }^{47}$

De ce fait qu'll est le Dieu vivant, véritablement homme et Dieu, la théologie, en tant que champ de foi et science de Dieu, montre aussi le visage d'un homme. Et pour qu'elle soit aussi une théologie authentique, elle doit, de façon vraiment nécessaire et pas seulement accessoire, bien montrer, dans son accomplissement le plus intime, une théologie qui exalte l'homme et qui, en conséquence, nous révèle ce Dieu qui se communique avec nous. Or, comme ce que Dieu nous donne, ce n'est pas en fin de compte son don créé mais Luimême, c'est le Dieu éternel même qui vient à l'homme, qui par sa grâce agit en cet homme, en sorte que celuici ouvre librement son cœur de petite créature, pénètre toute sa splendeur et l'immensité de vie de Dieu.

Cela dit, l'homme est toujours invité à chercher le fondement de son être et de sa liberté par-delà l'horizon des certitudes courtes. Ainsi, l'être humain, doit être guidé par une espérance invincible et constitutive, de telle sorte que seul un acte de foi peut l'engager en certaines voies, puisque l'homme ne peut pas tout vérifier par lui-même et à tout instant - reconnaît Gesché. II doit donc renoncer à ses prétensions et à ses illusions de pouvoir tout contrôler par lui-même, parce que, au fond, l'homme avance en raisonnant, mais il avance aussi en croyant. Ce que cette force vive de la

\footnotetext{
${ }^{47}$ RAHNER, K. Marie mère du Seigneur, p. 37
}

foi délivre et libère en nous, selon Rahner, c'est notre capacité de nous ouvrir et de nous déterminer au-delà du seul vérifiable, pour oser tenter notre aventure humaine de nous définir au plus haut de nous-mêmes, d'inscrire le fini dans l'Infini.

De plus, "pour une christologie, d'un point de vue humain, et donc aussi d'un point de vue de théologie fondamentale, il est légitime de partir de la relation qui existe de fait entre le chrétien croyant et Jésus Christ ". ${ }^{48}$ C'est pourquoi, pour Rahner, celui qui laisse Jésus lui dire la vérité ultime de sa vie, et qui confesse que dans Jésus et dans sa mort Dieu lui a dit la parole ultime, celui-là accueille Jésus comme le Fils de Dieu, tel que l'Église le confesse, quelle que soit la façon dont résonne pour lui-même la conceptualité mal venue en théorie, par laquelle est formulé le déploiement croyant de son existence. D'après lui, celui qui assume son existence, et donc son humanité, en silencieuse patience, (dans la foi, l'espérance et l'amour), qui l'assume comme le mystère qui se cache au mystère de l'amour éternel et porte la vie dans le sein de la mort, celui-là - même s'il ne le sait pas - dit oui au Christ.

Voilà le parcours! Celui qui assume son être homme et travaille son devenir homme, en se laissant façonner par le Christ, celui-là a accueilli le Fils de l'Homme, parce qu'en lui Dieu a assumé l'homme. C'est ainsi que le devenir-homme du Fils de Dieu pousse le devenir homme de chacun de nous, car Dieu Lui-même est devenu homme dans la chair de Jésus de Nazareth, et s'est fait notre prochain. L'Unique est devenu à la fois le proche et le lointain, celui qui doit être à la fois accueilli et aimé. Effectivement, toute la Loi et les Prophètes trouvent leur accomplissement dans cette perfection d'amour qui déborde et qui dépasse toutes les limites humaines quand nous nous disposons à écouter l'énonciation de Celui qui nous a aimés le premier.

C'est ainsi que la nature humaine devient, par l'Incarnation du Fils, l'énonciation du Logos en devenir, objet de la connaissance et de la puissance créatrice de Dieu au fond de chacun de nous. Quand cela arrive, naît justement ce que nous pouvons appeler une nature humaine transfigurée: le Fils est à la fois l'image parfaite du Père et de l'homme. II est l'homme possible. II rend l'homme toujours possible, parce qu'il se pose et il s'exprime ainsi lui-même. II met en évidence un caractère d'ouverture relationnelle radicale et il nous invite à faire de même dans nos vies.

\section{ili. Conclusion}

L'échange de parole du Dieu Trinité se laisse écouter dans le monde. Le Père n'est pas sans le Fils : «Je ne suis pas seul, dit Jésus, le Père est avec moi »

${ }^{48}$ RAHNER, K. « Jésus Christ ». Dans: Traité fondamental de la foi, p. 232. 
(Jn 16, 32), de même qu'll n'est pas sans l'homme, car "ll nous a choisis en Christ avant la fondation du monde » (cf. Ep 1, 4). L'aventure de Dieu avec les hommes se fait entendre, et nous voici renvoyés à l'histoire de la Révélation qui nous montre comment le Père, le Fils et l'Esprit Saint travaillent conjointement pour le salut du monde. La Parole se manifeste ainsi dans la multiplicité des gestes d'amour communiqués à la Création depuis le commencement et cette communication atteint l'événement majeur de la relation de Dieu avec nous, son autocommunication, par l'acte irréversible de l'Incarnation du Logos divin - son devenir-homme dans le monde.

En effet, la Parole est tout près de nous, sur nos lèvres et dans nos cœurs (cf. Rm 10, 8) et quand Dieu se révèle à l'homme, quand II lui dit une parole divine, il faut que cette communication se produise dans une parole humaine. Dieu précisément ne peut parler à l'homme qu'avec des paroles que l'homme puisse comprendre et qui soient par conséquent des paroles humaines. Le rapport entre la Parole de Dieu et la parole de l'homme dans la parole de la Révélation, comme nous l'avons vu, est ainsi multiple et à tel point que si la Parole de Dieu ne se liait pas à une parole humaine, elle ne dirait rien à l'homme. Selon Rahner, c'est dans cette impuissance qu'elle acquiert un quelque chose de plus : elle devient réalité dans le milieu de son autre, elle devient de fait la réalité de son autre.

C'est justement dans ce processus que se produit le devenir-homme du Fils de Dieu et le devenir homme de l'homme, du fait que la révélation trinitaire est la voix qui s'énonce, puisqu'elle découvre à chacun de nous notre propre mystère. L'expérience du Logos venant dans le monde est ainsi l'expérience de chaque être humain appelé, lui aussi, à sortir de soi pour s'accomplir dans l'autre, à risquer de se perdre en allant à la rencontre des autres. La doctrine trinitaire annonce donc son intelligibilité dans cette expérience de la personne humaine saisie par la personne divine dans le même carrefour où se croisent l'anthropologie et la christologie. De fait, seule une grammaire d'un autoénoncé possible de Dieu, comme dit Rahner, pourrait dresser la carte de ces relations et articuler pour l'être humain le terrain où il fonde sa relation avec Dieu pour féconder en même temps sa relation avec le monde créé et avec les autres créatures et les autres hommes, ses frères.

Une "grammaire de relations", comme celle évoquée par Rahner et annoncée par Joseph Moingt, puisque l'Être en devenir révèle un Dieu qui parle et« il ne parlerait pas à travers l'opacité de la création s'il ne parlait en lui-même, non à soi, d'une parole monologuante qui ne pourrait que l'enfermer dans sa solitude, mais à d'autres lui-même, d'une vraie parole dialoguante qui est l'extraposition d'un Je et d'un Tu ».49

\footnotetext{
${ }^{49}$ MOINGT, J. « Dieu qui vient à l'homme, t. II-2 », p. 190.
}

C'est ainsi qu'll nous adresse sa Parole et nous invite à y répondre avec générosité. Son Logos est appel et envoi, car avec lui le Père appelle son Fils à l'existence et l'envoie dans le temps. Ce circuit de langage qui appelle et envoie dévoile par l'acte de communication un Dieu avec nous. Le «pour nous » de Dieu le Père qui nous livre son Fils n'est donc pas pure condescendance, il appartient à son Être en tant qu'll est Amour. C'est sa «manière d'être " en relation au monde par et dans son Verbe et son Esprit.

Telle est l'identité de Dieu Trinité, une communauté ouverte aux relations, telle est notre identité. Tel est le devenir-homme du Fils de Dieu, l'énonciation d'un langage intersubjectif qui unifie, tel est notre appel et notre envoi : devenir hommes unifiés et bien intégrés dans le monde, champ d'humanisation où Dieu advient pour nous, en nous et avec nous. Pour cela, Jésus lui-même nous appelle à porter notre regard sur son intimité avec Dieu, sur sa « manière d'être " pour Dieu, en Dieu et avec Dieu, quand il dit: "que tous soient un comme toi, Père, tu es en moi et moi en toi », afin que s'accomplisse notre salut: "qu'ils soient un comme nous sommes un, moi en eux et toi en moi, qu'ils se trouvent accomplis dans l'unité » (cf. Jn 17, 21-23).

Conformer le nous humain au nous divin est donc par excellence le contenu de la Révélation. Cela nous remplit d'espérance "et l'espérance ne déçoit point, parce que l'amour de Dieu a été répandu dans nos cœurs par le Saint Esprit qui nous fut donné » (Rm 5, 5).De même, l'Esprit nous donne la force, car Lui, qui est au-delà de toute connaissance et de tout entendement, Lui l'indicible (1 P 1, 8), l'inexprimable, l'ineffable (2 Co 9, 15), pour nous rendre à notre tour image de Dieu, est devenu Lui-même, par amour des hommes, Image du Dieu invisible (Col 1, 15), de manière à prendre forme en nous, pour que nous, à notre tour, nous nous transformions, jusqu'à devenir ce que nous étions à l'origine.

$\mathrm{Au}$ fond, la profondeur du devenir homme à notre tour à l'issue du devenir-homme du Fils de Dieu est inépuisable. Son dynamisme nous entraîne dans un mouvement éternel de désir qui répond à l'image d'une source, comme celle évoquée par Balthasar : bien que l'image soit bonne, elle ne suffira jamais. Elle ne révélera que la "surface » de l'eau, son jaillissement reste sans fin et à chaque fois il est encore plus profond. À vrai dire, chemin faisant, peu importe le point où nous en sommes, nous resterons toujours en marche et toujours au commencement. Pourtant, cette image nous console. Nous serons toujours accompagnés, car : «Au commencement était le Logos, et le Logos était auprès de Dieu, et le Logos était Dieu » (Jn 1, 1).

Supposons que quelqu'un se tienne debout, près d'une source. II admettra ce jaillissement sans fin qui toujours monte de l'intérieur et se verse en dehors, il ne dira cependant jamais qu'il a vu l'eau entière. Car comment 
verrait-il celle qui est encore cachée dans le sein de la terre? Et même s'il restait très longtemps à côté de cette ébullition, il serait toujours au commencement de sa vision de l'eau. Car jamais l'eau ne se lasse de couler et sans cesse elle recommence à sourdre. II en est de même de celui qui regarde vers cette beauté divine et infinie : comme ce qu'à chaque instant il trouve est toujours plus nouveau et plus paradoxal que ce que sa vue avait déjà saisi, il ne peut qu'admirer ce qui, à chaque instant, se présente à lui, mais son désir de regarder ne se fatigue jamais, car les révélations auxquelles il s'attend seront toujours plus magnifiques et plus divines que tout ce qu'il a déjà vu. ${ }^{50}$

\section{Références Bibliographiques}

1. BALthASAR, U. H. von. Présence et pensée. Essai sur la philosophie religieuse de Grégoire de Nysse. Paris : Beauchesne, 1988, pp. 101-139.

2. DELEUZE, G. Pourparlers. Paris : Minuit, 1990.

3. DOCUMENTS DE VATICAN ॥ (Constitution Dogmatique sur la Révélation Divine).

4. GESCHÉ, A. «Topiques de la question du salut ». Dans : Dieu pour penser V. La destinée. Paris : Cerf, 1995, pp. 27-70.

5. GRÉGOIRE DE NYSSE. «Incarnation et œuvre du salut ». Dans : Discours catéchétique. SC 453, 2000, pp. 203-235.

$6 . \quad$. "Traité de la perfection». Dans: Écrits spirituels. Le Pères dans la foi. Paris: Migne, 1990, pp. 30-60.

7. HEGEL, G. «La science de la logique». Dans : Encyclopédie des sciences philosophiques. Paris: Librairie Philosophique J.Vrin, vol. 1. 1970.

8. JACQUES, F. "La parole et l'écriture ». Dans : L'arbre du texte et ses possibles. Paris: Librairie Philosophique J.Vrin, 2007.

9. JEANNIĖRE, A. «En archê ên O Logos ». Dans : RSR, 2/1995 (Tome 83), pp. 241-247.

10. KASPER, W. «Fondement de la doctrine trinitaire». Dans: Le Dieu des chrétiens. Paris: CERF, 1985, pp. 337-380.

11. LÉON-DUFOUR, X. Lecture de l'évangile selon Jean, t. I (Parole de Dieu). Paris : Seuil, 1988.

12. MOINGT, J. «Dieu qui vient à I'homme, t. II-2: De l'apparition à la naissance de Dieu - 1. Apparition ». Dans : Cogitatio Fidei, 245. Paris : CERF, 2005.

13. . "L'homme qui venait de Dieu». Dans: Cogitatio Fidei, 176. Paris : CERF, 1999.

14. RAHNER, K. "Jésus Christ». Dans: Traité fondamental de la foi. Paris: Centurion, 1983, pp. 203-258.

15. _. "Le traité dogmatique 'De Trinitate' ». Dans : Écrits théologiques, VIII. Paris : DDB, 1967, pp. 107140.

\footnotetext{
${ }^{50}$ In Cant. H. 11; I, 1000 AB ; cf. Didyme l'Aveugle. P. G., 39, 1649 C. (Cité par: BALTHASAR, U. H. von. Présence et pensée. Essai sur la philosophie religieuse de Grégoire de Nysse. Paris: Beauchesne, 1942, pp. 124-125).
}

16. Marie mère du Seigneur. Paris : Éditions de l'Orante, 1960.

17. _ "Méditation 12: L'Incarnation de Dieu ». Dans: L'Esprit Ignatien. (Sous la direction de Michel Fédou). Paris : CERF, 2016, pp. 163-180.

18. SESBOÜÉ, B. "Le sauver et le salut. Problématique ». Dans: Jésus-Christ l'unique Médiateur. Essai sur la rédemption et le salut. Paris : Desclée, 1988, pp. 1-120.

19. SIMOENS, Y. Selon Jean. 2. Une interprétation. Bruxelles: Éditions de l'Institut d'Études Théologiques, 1997, pp. 29-77.

20. TAVARD, G. «Le discours ». Dans : La Vision de la Trinité. Paris : CERF, 1989, pp. 135-160.

21. THEOBALD, C. Croire aujourd'hui en Jésus, Christ et Saint de Dieu, 2016. (Cours de Christologie Polycopié).

$22 . \quad$. ¿La réception des Écritures inspirées 》. Dans : RSR, 4/2005 (Tome 93), pp. 545-570.

23. ZOURABICHVILI, $F$. Conférence prononcée à Horlieu (Lyon) le 27 mars 1997. "Qu'est-ce qu'un devenir, pour Gilles Deleuze? » Document accessible à l'adresse : horlieu-editions.com/ brochures/zourabichvili-qu-est-ce-qu-un-devenirpour-gilles-deleuze.pdf 